\title{
Microtubule-driven nuclear rotations promote meiotic chromosome dynamics
}

Nicolas Christophorou ${ }^{1,2,5}$, Thomas Rubin ${ }^{1,2,5}$, Isabelle Bonnet ${ }^{3,4}$, Tristan Piolot ${ }^{1,2}$, Marion Arnaud $^{1,2}$ and Jean-René Huynh ${ }^{1,2^{*}}$

1: Department of Genetics and Developmental Biology, Institut Curie, Paris, France

2: CNRS UMR3215; Inserm U934 F-75248 Paris, France

3: Laboratoire Physico-Chimie, Institut Curie, Paris, France

4: CNRS UMR 168, UPMC, F-75248 Paris, France

5: These authors contributed equally

*Corresponding author: jean-rene.huynh@curie.fr

Keywords: meiosis, pairing, microtubules, Dynein, SUN and KASH domain proteins, Mud, Drosophila, germline, oogenesis. 


\section{ABSTRACT}

At meiosis onset, each chromosome needs to find its homologue and pair to ensure proper segregation. In Drosophila, pairing occurs during the mitotic cycles preceding meiosis. Here we show that germ cell nuclei undergo dramatic movements during this developmental window. We demonstrate that microtubules and Dynein are driving nuclear rotations and are required for centromere pairing and clustering. We further found that Klaroid (SUN) and Klarsicht (KASH) colocalize with centromeres at the nuclear envelope and are required for proper chromosome motions and pairing. We identified Mud (NuMA in vertebrates) as colocalizing with centromeres, Klarsicht and Klaroid. Mud is also required to maintain the integrity of the nuclear envelope and for the correct assembly of the synaptonemal complex. Our findings reveal a mechanism for chromosome pairing in Drosophila, and indicate that microtubules, centrosomes and associated proteins play a crucial role in the dynamic organization of chromosomes inside the nucleus. 


\section{INTRODUCTION}

One central event at the onset of meiosis is the pairing of homologous chromosomes. This seemingly simple process requires complex mechanisms as homologous chromosomes need to find each other, align along their length and assess their homology before pairing ${ }^{1-2}$. Pairing is then reinforced by synapsis, i.e. the assembly of the synaptonemal complex (SC) ${ }^{3}$. Synapsis is often followed by recombination events, which ensure exchange of parental genetic information and segregation of homologous chromosomes during the first meiotic anaphase. Although great progress has been made in recent years, uncovering the molecular mechanisms that promote homologous pairing has proven very challenging ${ }^{4-5}$. One reason is the exciting but bewildering diversity of mechanisms leading to pairing in different organisms $^{6}$. For example, the starting sites of pairing and synapsis are the telomeres in mammals ${ }^{7-8}$, there are specific sequences defining pairing centers for each chromosome in $C$. elegans ${ }^{9}$, while it was recently found that it starts at centromeres in Drosophila ${ }^{4-5,10-11}$. Meiotic chromosomes are further organized into telomere bouquet in yeast and mammals or clusters of centromeres in flies, whereby telomeres or centromeres aggregate on one side of the nucleus ${ }^{12-14}$. All of these different chromosome organizations imply many dynamic chromosome movements. Deciphering the successive steps of meiotic chromosome dynamics by live-imaging microscopy has been another challenge, especially in multicellular organisms. Only recently, cutting-edge time-lapse microscopy has allowed describing rapid Dynein-dependent movements of pairing centers in C. elegans, and rotational movements of telomeres in mouse spermatocytes ${ }^{15-19}$. These mechanisms are still very different from the actin-dependent telomere movements in budding yeast and the microtubule-driven horsetail

motions described in fission yeast ${ }^{20-21}$. Although setting chromosomes in motion to facilitate pairing seems a common theme, it is not possible to extrapolate the underlying mechanisms from one species to another ${ }^{6}$. 
In Drosophila, the pairing of meiotic chromosomes remains practically unexplored ${ }^{22}$. One reason is the long held assumption that meiotic pairing was the passive inheritance of a phenomenon called "somatic pairing", in which homologous chromosomes are always paired in somatic and germline cells. Meiotic pairing was thus viewed as an extension of a preexisting somatic pairing ${ }^{22}$. We and others have shown recently that it is not the case for autosomal chromosomes in germline stem cells and that homologous chromosomes are actively pairing in the mitotic region preceding the entry in meiosis ${ }^{23-24}$. These events take place in a specialized structure called the germarium at the anterior tip of each ovary (Figure 1A). The germarium is itself organized in several functional regions ${ }^{25}$. The mitotic region, also called region 1, is at the anterior tip of the germarium. There, germline stem cells (GSCs) divide asymmetrically to produce a new GSC and a cystoblast, which undergoes exactly four mitotic divisions to form a germline cyst made of 16 cells. These divisions are incomplete ${ }^{26}$. All 16 cells thus remain connected by ring canals and by a germline-specific organelle called the fusome made of ER-derived vesicles. The branched shape of the fusome is a useful marker to distinguish each stage, GSC, $\mathrm{CB}, 2-, 4$-, 8- and 16-cell cyst (cc) in the mitotic zone. We showed that pairing between autosomal homologues mostly occurs during the 4-cell and 8-cell cysts ${ }^{23-24}$. In 16-cell cysts, paired centromeres of different chromosomes start to aggregate into one or two clusters at the nuclear envelope ${ }^{10-11}$. After the last mitosis, all 16 cells enter prophase I of meiosis in the meiotic zone of the germarium, also called region 2, and build some segments of SC. However, only one cell, the future oocyte, will remain in meiosis, while the 15 other cells exit meiosis, endoreplicate their DNA and later become polyploid nurse cells.

In this study, we have improved further our technique of imaging live the germarium ${ }^{27}$ to investigate two main questions: 1) Are there chromosomal movements during meiotic 
pairing in Drosophila? and 2) What are the molecular mechanisms driving homologous pairing in pre-meiotic germ cells?

\section{$\underline{\text { RESULTS }}$}

\section{1) Dynamic movements of centromeres and rolling nuclei in 8-cell cysts}

While analyzing the pairing and clustering of centromeres in pre-meiotic germ cells ${ }^{23}$, we noticed that some centromeres showed coordinated and directional movements within specific nuclei. We followed each centromere with a CID-RFP fusion transgene and we labelled the fusome with a GFP-tagged marker to identify each stage of differentiation from GSCs to 16-

cell cysts ${ }^{28-29}$. In GSCs, CBs, 2-cell cysts and 4-cysts, we found that most centromeres moved independently from each other and covered a small nuclear volume (Figure 1B, 1C, 1E, Supplementary Video 1, Supplementary Fig. 1A, 1B; and see methods section for quantification of centromere trajectories). In contrast, in 8-cell cysts, about $45 \%$ of centromeres showed coordinated circular movements (or revolutions) covering most of the nuclear space (Figure 1B, 1D, 1F, Supplementary Video 1). Some centromeres could even undergo complete revolutions during one period of recording (Figure $1 \mathrm{G}, 1 \mathrm{H}$ ). These highly dynamic and directed movements were however transient as 16-cell cysts showed small covered volumes similar to 4-cell cysts (Figure $1 \mathrm{G}$ and $1 \mathrm{H}$ ).

We thus wondered whether the coordinated movements of centromeres were due to rotations of the entire NE or whether centromeres were moving independently of the NE. When we could detect circular motions of centromeres, we found similar and coordinated movements of nucleoporin foci on the NE (Supplementary Video 2). We concluded that the entire nuclear membrane was rotating. To test whether the entire nucleus, including all chromosomes, was also rotating, we made a Histone2A fused with a photoconvertible Dendra2 tag. This allowed us to label any sub-regions of chromatin in red with a brief pulse 
of a 405nm laser (Figure 1I). By visualizing simultaneously centromeres and labelled chromatin spots, we found that both were moving in the same direction with the same speed (Figure 1I and Supplementary Video 3). We concluded that each nucleus was rotating as a unit during a specific developmental window.

\section{2) Microtubules are required for centromere dynamics}

What could drive these nuclear rotations? Based on studies done in C. elegans, S. pombe, maize and mouse, we reasoned that the microtubule cytoskeleton was a good candidate ${ }^{15,17-}$ 18, 30-31. We first examined in more details the organization of microtubules in region 1 germline cysts. Using a GFP knock-in at an endogenous microtubule-associated protein (Jupiter) for live-imaging, we found that the microtubule cytoskeleton was mainly organized around the fusome as previously published (Figure 2A, Supplementary Video 4$)^{32-33}$. We also observed microtubules around the nuclear envelope and emanating from centrosomes (Figure 2A). Live-imaging also allowed us observing whip-like movement of microtubules in the cytoplasm (Supplementary Video 4). To decipher which structures were nucleating microtubules, we set up experimental conditions of live-imaging where we could both inactivate and activate the microtubule cytoskeleton. We fed young female flies with the microtubule polymerizing inhibitor colcemid during 4 hours and dissected the ovaries immediately. We found that germ cell microtubules were mostly depolymerized in such conditions and mitoses were arrested (Supplementary Video 5). Knowing that colcemid can be inactivated by UV light ${ }^{34}$, we found that a 5 sec UV pulse was sufficient to induce immediate re-growth of microtubules and rescue of mitoses (Supplementary Video 5). Imaging germline cysts immediately after UV pulse, we found microtubules growing from the fusome, centrosomes and nuclear envelops (Figure 2B, Supplementary Video 6). We 
concluded that all three structures can nucleate microtubules, although most microtubules localize along the fusome.

During these recordings, we also noticed that centrosomes displayed circular movements mainly in 8-cell cysts reminiscent of nuclear rotations (Supplementary Video 7). At this stage, $35 \%$ of centrosomes showed these rotations, while $65 \%$ remained static (Figure 2C), which was similar to centromere behavior (45\% dynamic and 55\% static). We thus imaged both centromeres and centrosomes simultaneously (using Cid::RFP and asterless::YFP, respectively) and found that the majority of dynamic centrosomes underwent revolutions in the same direction and with the same speed as centromeres of the same cell ( $87 \%$ of co-rotation; Figure 2D, 2G). However, during these co-rotations, centromeres and centrosomes were not co-localized at the nuclear envelop (28\% of dynamic co-localization; Figure 2E). In contrast, we found that in static nuclei, centromeres and centrosomes often colocalized (Figure 2F); and were associated with the fusome, as we and others had published for centrosomes ${ }^{32-33,35}$.

In the presence of colcemid both centromere movements and relative covered volumes were dramatically reduced in 8-cell cysts (Figure 2H, 2J, 2K and Supplementary Video 8). Furthermore, upon UV irradiation, the coordinated and directional movements of centromeres were immediately restored (Figure 2I, 2J', Supplementary Video 9). We made similar observations for centrosome movements (Figure 2B, Supplementary Video 10). In contrast, we did not observe any change in centromere dynamics in GSCs before and after the UV pulse (Supplementary Fig. 2 and Supplementary Video 11). We concluded that centromere movements in 8-cell cysts depend on microtubules.

\section{3) Centrosomes and Dynein are required for centromere dynamics}


We used the fact Drosophila germ cells can develop without centrosomes to assay centromere dynamics in absence of centrosomes ${ }^{36}$. We removed centrosomes genetically by knocking down sas-4 and asterless (asl), which are required for centriole duplication ${ }^{37-38}$. We found that in both sas-4_shRNA and asl_shRNA mutant 8-cell cysts (TRiP collection ${ }^{39}$ ), centromeres were still revolving but at a lower speed compared to control flies expressing a shRNA against white (3A-C; 3E-G and Supplementary Videos 12, 13 and 14). As a result, the relative covered volume per second was significantly reduced in knock-down conditions (Figure 3I). We concluded that centrosomes were required for efficient nuclear rotations, but were not the main driving force.

Dynein is a minus-end directed motor associated with microtubules. To test for a potential function of Dynein in driving nuclear rotations, we used two independent transgenic lines expressing shRNA targeting dynein in germ cells (Figure 3D, 3H and Supplementary Video 15). We found that the movement of centromeres displayed a dramatic reduction in the nuclear volume covered and no rotational movements were seen in 8-cell cysts expressing nanos:Gal4;UAS:Dhc_shRNA (Figure 3D, 3H, 3I and Supplementary Video 14). We confirmed these defects by using a viable but sterile combination of dynein alleles, $D h c 64 c^{3-}$ ${ }^{2} / D h c 64 c^{6-12}$, in which we never observed any rotation (Figure 3I and Supplementary Video 16). We further noticed that whip-like movements of microtubules were strongly reduced in this mutant condition (Supplementary Video 17 compared to Video 4 for wild type condition). We concluded that microtubules and Dynein played a crucial role in generating nuclear rotations in pre-meiotic germ cells.

\section{4) Microtubules, Centrosomes and Dynein are required for centromere pairing, clustering and homologous chromosome synapsis}


We previously showed that centromere pairing and clustering occurred most prominently at the 8 -cell cyst stage, which is when we detected most nuclear rotations ${ }^{23}$. We wanted to test if there was a functional connection between these two events. We thus assayed the effect of reducing microtubules, centrosomes and Dynein activity on centromere pairing and clustering, and on the formation of the synaptonemal complex (SC). Drosophila diploid cells have 4 pairs of homologues thus eight chromosomes. Therefore when all homologues are paired, 4 dots of CID can be distinguished. More than 4 dots are seen when not all centromeres are paired, and when centromeres become clustered, one should see only 1 or 2 dots ${ }^{10-11}$. In wild type 8-cell cysts nuclei, we counted an average number of CID foci of $3.8 \pm 0.8$ (Figure 4A, 4F) indicating that most chromosomes were paired and started to cluster at their centromeres. In germarium treated with colcemid, we observed a significant increase in CID foci with an average of $4.7 \pm 1.5$ (Figure 4B, 4F). Similarly, in the absence of centrosomes, the number of CID foci was increased to $4.7 \pm 1.6$ in sas -4 mutant and $4.8 \pm 1.2$ in asl mutant (Figure 4C, 4D, 4F). In germarium expressing nanos:Gal4;UAS:Dhc_shRNA, we observed an even stronger increase in CID foci with an average of 6.0 1.7 (Figure 4E, 4F).

We then investigated whether centromere clustering and synaptonemal complex assembly were affected in meiotic nuclei of region $2 \mathrm{a}$. We found that wild type pachytene nuclei displayed an average number of 2.0 $\pm 0.6 \mathrm{CID}$ foci per nucleus (Fig 4F, 4G). In the absence of centrosomes, we noticed a minor increase in the number of CID dots, $2.2 \pm 0.9$ and $2.6 \pm 1$ in sas-4 and asl mutant flies respectively (Fig 4F). We observed a much stronger phenotype in the presence of colcemid (4.4 \pm 2.4 dots) and when Dynein activity was disrupted either by RNAi (3.2 \pm 0.9 dots) or using viable but sterile combination of dynein alleles, $D h c 64 c^{3-2} / D h c 64 c^{6-12}$, with an average of $5.1 \pm 1.2$ dots of CID (Figure 4F). However, we noticed that even after long exposures to colcemid, centromeres managed to pair later during oogenesis, in region $2 \mathrm{~b}$ (Supplementary Fig 3). 
We found that in the presence of colcemid and in both nanos $>D h c_{-}$shRNA and $D h c 64 c^{3-2} / D h c 64 c^{6-12}$ flies, the SC of all meiotic nuclei appeared fragmented with shorter filaments (Figure 4H, 4K). We measured the total length of SC fragments in these three conditions and found that the length per cell was reduced by half compared to wild type (Figure 4L). In sas-4 and asl mutant ovaries, the phenotype was much less dramatic. The total SC length per cell was nonetheless slightly shorter than in wild type conditions and the normalized intensity was reduced (Figure 4L and Figure 6J).

We concluded that centrosomes play a secondary role in promoting pre-meiotic pairing, centromere clustering and SC formation, which correlates with inefficient but existing nuclear rotations in sas-4 and asl knock-downs. In contrast, in absence of dynamic microtubules or Dynein motor, and thus, in complete absence of nuclear rotations, pre-meiotic pairing, centromere clustering and synapsis between homologues are strongly affected.

\section{5) SUN and KASH domain proteins Klaroid and Klarsicht are required for centromere dynamics, pairing and synapsis}

SUN and KASH domain proteins are transmembrane proteins localizing at the inner and outer nuclear membranes respectively, and form bridges between the inside of the nucleus and the cytoplasmic cytoskeleton in a wide range of cells ${ }^{40-43}$. In Drosophila, two genes encode for SUN-domain proteins, Klaroid and Spag4, and two genes encode for KASH-domain proteins, Klarsicht and MSP-300 ${ }^{44-48}$. Spag4 is expressed specifically in male testis ${ }^{45}$, while MSP-300 interacts with actin rather than microtubules ${ }^{48-49}$. We thus decided to investigate the function of klaroid (koi) and klarsicht (klar) during early female meiosis.

As previously published, we found that both Klar and Koi had a homogeneous perinuclear localization across all stages in germline and somatic nuclei (Supplementary Fig. 4) ${ }^{46}$. However, in region 1 germ cells, both Klar and Koi formed dots at the NE (Figure 5). 
Interestingly, these dots often colocalized or were in close proximity to centromeres in fixed and live ovaries (Figure 5Aa-5Ad, 5B, Supplementary Video 18). We observed these colocalizations mostly in 8-cell cysts, but also in some 4-cell and 16-cell cysts (Supplementary Fig. 4).

Using live imaging, we found that centromere motions were reduced and the relative covered volume per second was also significantly decreased in 8-cell cysts mutant for null alleles of klaroid and klarsicht, koi $^{80}$ and klar $^{\text {marb-CD4 }}$ respectively ${ }^{44,50}$ (Figure $5 \mathrm{C}-\mathrm{G}$ and Supplementary Video 19-20). Some centromere foci still displayed circular movements but at a much lower speed thus the volume covered per second was low. These movements were more affected in $k l a r^{\text {marb-CD4 }}$ than in $k o i^{80}$ mutant germ cells (Figure 5G, Supplementary Figure 5).

We then investigated the effects of klarsicht and klaroid loss of function on pre-meiotic centromere pairing, clustering and synapsis. Centromere pairing was significantly affected in $k^{\text {karb-CD4 }}{ }^{\text {moi }}{ }^{80}$ and klar $^{\text {marb-CD4 }}, k^{80}{ }^{80}$ double mutant ovaries (Figure 6A-6D, and Supplementary Table 1). Clustering of centromeres in region 2a pachytene nuclei was also disrupted, but much less dramatically (Figure 6D). We found that most klar ${ }^{\text {marb-CD4 }}$ and klar $^{\text {marb-CD4 }}, \mathrm{kol}^{80}$ mutant ovaries had reduced level of SC, indicating defects in synapsis (Figure 6F, 6H, 6I and 6J). In contrast, most pachytene nuclei mutant for klaroid $^{80}$ showed a normal SC (Figure 6G, 6I and 6J). In addition, we noticed in some klar; koi double mutant germarium the presence of large aggregates of SC instead of the typical filamentous threadlike structure (7.3\%, n=151, Figure 6J, 6K, Supplementary Fig. 6). These aggregates were reminiscent of the polycomplexes (PCs) described by Jeffress and colleagues ${ }^{51}$. Overall, we concluded that the KASH domain protein Klarsicht and the SUN domain protein Klaroid were essential for centromere motions and pairing, and also played an important function in SC assembly. 


\section{6) The Dynein-interacting protein Mud co-localizes with Klarsicht/Klaroid and is required for synapsis between homologues}

Next, we searched for proteins directly interacting with Dynein and the nuclear envelope, which could play a role during meiosis. We focused on Mud (Mushroom body defect), the Drosophila homologue of NuMA, for several reasons: 1) Vertebrate NuMA is known to interact directly with Dynein to assemble the mitotic spindle, while its Drosophila and C. elegans homologues interact with Dynein to position the spindle in neuroblasts and the onecell stage embryo ${ }^{52-56}$; 2) mud null alleles are viable but females are sterile and males are fertile, indicating a specific requirement for mud in female germ cell development; 3) Mud is an essential component of the meiosis II spindle in Drosophila oocyte ${ }^{57}$; and 4) Mud is expressed in the germarium and localizes to the nuclear envelope ${ }^{57}$ (Figure 7A and 8F).

In addition to this localization, we noticed dots of Mud on the cytoplasmic side of the $\mathrm{NE}$ and found that these dots were precisely juxtaposed to centromeres (Figure 7B and 7C), and could co-localize with Klarsicht and Klaroid (Figure 7Da-7Dd and 7Ea-7Ed). We then analysed germaria mutant for mud to investigate a potential function in region 1 and 2 . We

found by live imaging that the $m u d^{f 01205}$ mutation did not disturb significantly centromere dynamics (Figure 7G, 7I and 7J, Supplementary Video 21 and 22). Centromeres pairing and clustering were only slightly affected in $m u d^{f 01205}$ germaria (Figure $8 \mathrm{~B}, 8 \mathrm{C}$ ). However, we detected significant genetic interactions between mud and both klarsicht and klaroid for centromere pairing, but not for centromere clustering (Figure 8C, Supplementary Table 2 and $3)$.

Next, we examined the assembly of the SC in mud $^{f 01205}$ mutant germarium by analyzing the localization of $\mathrm{C}(3) \mathrm{G}$. We found that $82 \%$ of mutant germarium and around 90\% of germarium expressing mud_shRNAi had a reduction of fluorescence ranging from 30- 
$40 \%$ for the shRNAi and 50\% for $m u d^{f 01205}$ (Figure 8D, 8E, 6I, 6J; and Supplementary Fig. 7).

Strikingly, we also found that $16 \%$ of und $^{f 01205}$ germarium formed polycomplexes (PCs) instead of thread-like SCs (Supplementary Fig. 5). These PCs could be found as early as region $2 \mathrm{a}$ and were still visible in later stages of oogenesis (Figure $7 \mathrm{H}$ and $7 \mathrm{I}$ ). We noticed that the presence of PCs correlated with an absence of nuclear envelope marked by Lamin and by Lectin (Figure 7G and 7H, Supplementary Fig. 8). DAPI staining also revealed that DNA was diffused in the oocyte cytoplasm (Figure 7H and 7I). Despite this nuclear phenotype, mutant oocytes were correctly determined and polarized, and grew properly into late stage egg chambers (Figure 7I).

We examined in more details the structure of polycomplexes using super-resolution microscopy. We used antibodies recognizing C(3)G and Corona (Cona), which are transverse filaments and central element components of the SC, respectively, and required for SC formation ${ }^{58-59}$. In wild type pachytene nuclei, we could see the central element protein Cona localized as a single line between two threads of $\mathrm{C}(3) \mathrm{G}$ labeling the edges of the transverse filament, as predicted from previous studies (Figure $7 \mathrm{~J}-\mathrm{J}{ }^{\prime}{ }^{59}$. In mud $^{\mathrm{f01205}}$ polycomplexes, we could also distinguish alternate threads of Cona and $\mathrm{C}(3) \mathrm{G}$, indicating the PCs were not simple aggregates of SC components as appeared with regular confocal microscopy (Figure 5K). Overall, we concluded that Mud played a minor in centromere dynamics and pairing, but was required for the integrity of the nuclear membrane and the assembly of the synaptonemal complex.

\section{DISCUSSION}

Rotations of nuclei have been described previously in somatic cells, their function

remains however unclear ${ }^{60-63}$. In germ cells, meiotic chromosome movements are thought to be required for homologues pairing, removing chromosome entanglements, promoting 
maturation of recombination intermediates, or for assessing chromosome homology before synapsis, in different model organisms ${ }^{64-65}$. In Drosophila, we found a high temporal correlation between nuclear rotations and chromosome pairing occurring mainly in 8-cell cysts. Our work uncovered a second interesting correlation between the speed of nuclear rotation and the degree of centromere pairing and clustering. Indeed, mutations in klaroid affected the least nuclear rotations and disrupted the least centromere associations and synapsis. Rotations were slowed down more significantly in klarsicht, sas-4 and asl mutant germ cells. Accordingly, we observed strong defects in the initial pairing of centromeres and in SC formation. Finally, nuclear rotations were completely abolished in the absence of Dynein or dynamic microtubules. In dynein mutant germ cells, we could distinguish an average of 6 centromeres during pre-meiotic pairing, which is higher than any mutants we have tested previously, including null alleles of $c(3) G^{23}$. Similarly, we counted 5 centromeres on average during clustering in region $2 \mathrm{a}$, a mutant phenotype that is comparable to the strongest ord or $c(3) G$ mutations (lateral and central elements of SC respectively) ${ }^{10-11}$. Nuclear rotations thus play an important role in homologue chromosome pairing and SC formation.

We found that microtubules could be nucleated from the fusome, the nuclear envelop and the centrosome in region 1 germ cells. Based on these observations and our centrosome mutant analysis, we can speculate that the whip-like movements of microtubules could be the main forces creating cytoplasmic flows, as observed in many biological systems and demonstrated theoretically ${ }^{66}$. In addition, microtubules nucleated by the centrosomes could also push on the nucleus and the cell membrane, which could bias nuclear movement toward one direction of rotation as proposed for the migration of this same oocyte nucleus later on during oogenesis ${ }^{67}$. These two forces depend on microtubules and dynein, and would act redundantly for efficient and unidirectional nuclear rotations. 
However, even in absence of dynamic microtubules, centromeres ended up paired, albeit much later in region 2b (Supplementary Fig. 3). Synapsis, on the other hand, was completely disrupted. We thus believe that like in yeast and worms, these movements are there to facilitate pairing, synapsis or recombination, but that at least chromosome pairing could occur slowly without motions by redundant mechanisms. In flies, Spag4, is a second SUN-domain protein, but it is only expressed in male testis and is thus not likely to play a role during oogenesis ${ }^{45}$. There is also a second KASH-domain protein called MSP-300/Nesprin, which interacts with the actin cytoskeleton ${ }^{48-49}$. In the absence of microtubules, we found that nuclei were not "rolling" anymore; however, we noticed that they still showed some back and forth "rocking" movements. It will be interesting to investigate whether MSP300/Nesprin and the actin cytoskeleton are involved in these rocking movements ${ }^{18,31}$.

We found that although mud mutant ovaries only showed mild defects in centromere dynamics, we were able to uncover significant genetic interactions with klaroid and klarsicht in this same process. Mud most striking features in our study were its co-localization with centromeres in interphasic germline cysts and the formation of polycomplexes in mud mutant cysts. The formation of PCs was associated with a lack of nuclear membrane and diffused DNA in the cytoplasm, suggesting that Mud is required to maintain the nuclear envelope integrity. We propose that the disappearance of the NE in mud cysts is the primary defect leading first to the de-localization of DNA into the cytoplasm and then the formation of PCs. PCs could thus be the result of self-assembly of SC components polymerizing in the absence of chromatin. We also observed PCs in klaroid and klarsicht mutants although at a lower penetrance than in mud mutants. Interestingly, large distortions of the NE were also observed in muscle cell nuclei mutant for unc-84, which encodes a C. elegans SUN protein ${ }^{68}$. These deformations were particularly strong in these cells, because muscle cell nuclei are subjected to mechanical stress. It is likely that rolling nuclei of 8-cell cysts are also exposed to some 
mechanical forces. Klarsicht, Klaroid and Mud may all participate in maintaining the integrity of the nuclear envelope in these conditions. In their absence, the NE is weakened and cannot resist mechanical forces, which also leads to SC assembly defects. In the most extreme cases the NE completely disappears causing the formation of PCs. Interestingly, Mud initially localizes at the NE of all germline cells in region 1, but then becomes localized only to the cells remaining in meiosis in region $2 \mathrm{a}$, and finally only specifically at the $\mathrm{NE}$ of the oocyte (Figure 7A and 8F). This may hint that the meiotic nucleus is subjected to specific mechanical forces during oogenesis.

\section{ACKNOWLEDGMENTS}

We wish to thank Flora Llense for observing that Mud colocalized with centromeres and Edith Heard for suggesting "rolling" nuclei. We are grateful to Siegfried Roth, Michael Welte, Janice Fischer and the Bloomington Stock center for flies and reagents. Conceived and designed the experiments: NC TR JRH. Performed the experiments: NC TR MA. Analyzed the data: NC TR JRH. IB conceived and performed centromere correlation analysis. TP performed SIM microscopy. Wrote the paper: JRH; NC. We acknowledged great technical support from the PICT@BDD imaging platform. This work was supported by the European Research Council (ERC EPIGENETIX N²50367). N.C. is supported by Institut Curie, FRM post-doctoral fellowship (SPF20111223331) and DEEP LabEx, T.R. is supported by an FRM Ingenieur Fellowship ( ${ }^{\circ}$ ING20140129247), J.R.H. lab is funded by CNRS, Ville de Paris, ANR and FSER (Schlumberger). 


\section{REFERENCES}

1. Bhalla, N. \& Dernburg, A.F. Prelude to a division. Annu Rev Cell Dev Biol 24, 397-424 (2008).

2. Lake, C.M. \& Hawley, R.S. The molecular control of meiotic chromosomal behavior: events in early meiotic prophase in Drosophila oocytes. Annu Rev Physiol 74, 425-451 (2012).

3. Page, S.L. \& Hawley, R.S. The genetics and molecular biology of the synaptonemal complex. Annu Rev Cell Dev Biol 20, 525-558 (2004).

4. $\quad$ Obeso, D., Pezza, R.J. \& Dawson, D. Couples, pairs, and clusters: mechanisms and implications of centromere associations in meiosis. Chromosoma 123, $43-55$ (2014).

5. Tsai, J.H. \& McKee, B.D. Homologous pairing and the role of pairing centers in meiosis. $J$ Cell Sci 124, 1955-1963 (2011).

6. Gerton, J.L. \& Hawley, R.S. Homologous chromosome interactions in meiosis: diversity amidst conservation. Nat Rev Genet 6, 477-487 (2005).

7. Boateng, K.A., Bellani, M.A., Gregoretti, I.V., Pratto, F. \& Camerini-Otero, R.D. Homologous pairing preceding SPO11-mediated double-strand breaks in mice. Dev Cell 24, 196-205 (2013).

8. Brown, P.W. et al. Meiotic synapsis proceeds from a limited number of subtelomeric sites in the human male. Am J Hum Genet 77, 556-566 (2005).

9. MacQueen, A.J. et al. Chromosome sites play dual roles to establish homologous synapsis during meiosis in C. elegans. Cell 123, 1037-1050 (2005).

10. Takeo, S., Lake, C.M., Morais-de-Sa, E., Sunkel, C.E. \& Hawley, R.S. Synaptonemal complex-dependent centromeric clustering and the initiation of synapsis in Drosophila oocytes. Curr Biol 21, 1845-1851 (2011).

11. Tanneti, N.S., Landy, K., Joyce, E.F. \& McKim, K.S. A pathway for synapsis initiation during zygotene in Drosophila oocytes. Curr Biol 21, 1852-1857 (2011).

12. Scherthan, H. A bouquet makes ends meet. Nat Rev Mol Cell Biol 2, 621-627 (2001).

13. Zickler, D. From early homologue recognition to synaptonemal complex formation. Chromosoma 115, 158-174 (2006).

14. Zickler, D. \& Kleckner, N. The leptotene-zygotene transition of meiosis. Annu Rev Genet 32, 619-697 (1998).

15. Baudrimont, A. et al. Leptotene/zygotene chromosome movement via the SUN/KASH protein bridge in Caenorhabditis elegans. PLoS Genet 6, e1001219 (2010).

16. Shibuya, H., Ishiguro, K. \& Watanabe, Y. The TRF1-binding protein TERB1 promotes chromosome movement and telomere rigidity in meiosis. Nat Cell Biol 16, 145-156 (2014).

17. Wynne, D.J., Rog, O., Carlton, P.M. \& Dernburg, A.F. Dynein-dependent processive chromosome motions promote homologous pairing in C. elegans meiosis. J Cell Biol 196, 4764 (2012).

18. Shibuya, H., Morimoto, A. \& Watanabe, Y. The dissection of meiotic chromosome movement in mice using an in vivo electroporation technique. PLoS Genet 10, e1004821 (2014).

19. Lee, C.Y. et al. Mechanism and regulation of rapid telomere prophase movements in mouse meiotic chromosomes. Cell Rep 11, 551-563 (2015).

20. Ding, D.Q., Chikashige, Y., Haraguchi, T. \& Hiraoka, Y. Oscillatory nuclear movement in fission yeast meiotic prophase is driven by astral microtubules, as revealed by continuous observation of chromosomes and microtubules in living cells. J Cell Sci 111 ( Pt 6), 701-712 (1998).

21. Trelles-Sticken, E., Adelfalk, C., Loidl, J. \& Scherthan, H. Meiotic telomere clustering requires actin for its formation and cohesin for its resolution. J Cell Biol 170, 213-223 (2005).

22. Cahoon, C.K. \& Hawley, R.S. Flies get a head start on meiosis. PLoS Genet 9, e1004051 (2013).

23. Christophorou, N., Rubin, T. \& Huynh, J.R. Synaptonemal complex components promote centromere pairing in pre-meiotic germ cells. PLoS Genet 9, e1004012 (2013). 
24. Joyce, E.F., Apostolopoulos, N., Beliveau, B.J. \& Wu, C.T. Germline progenitors escape the widespread phenomenon of homolog pairing during Drosophila development. PLoS Genet $\mathbf{9}$, e1004013 (2013).

25. Huynh, J.R. \& St Johnston, D. The origin of asymmetry: early polarisation of the Drosophila germline cyst and oocyte. Curr Biol 14, R438-449 (2004).

26. Mathieu, J. et al. Aurora B and cyclin B have opposite effects on the timing of cytokinesis abscission in Drosophila germ cells and in vertebrate somatic cells. Dev Cell 26, 250-265 (2013).

27. Fichelson, P. et al. Live-imaging of single stem cells within their niche reveals that a U3snoRNP component segregates asymmetrically and is required for self-renewal in Drosophila. Nat Cell Biol 11, 685-693 (2009).

28. Huynh, J.R., Shulman, J.M., Benton, R. \& St Johnston, D. PAR-1 is required for the maintenance of oocyte fate in Drosophila. Development 128, 1201-1209 (2001).

29. Schuh, M., Lehner, C.F. \& Heidmann, S. Incorporation of Drosophila CID/CENP-A and CENP-C into centromeres during early embryonic anaphase. Curr Biol 17, 237-243 (2007).

30. Yoshida, M. et al. Microtubule-organizing center formation at telomeres induces meiotic telomere clustering. J Cell Biol 200, 385-395 (2013).

31. Sheehan, M. \& Pawlowski, W.P. Live imaging of rapid chromosome movements in meiotic prophase I in maize. Proc Natl Acad Sci U S A 106, 20989-20994 (2009).

32. Bolivar, J. et al. Centrosome migration into the Drosophila oocyte is independent of BicD and egl, and of the organisation of the microtubule cytoskeleton. Development 128, 1889-1897 (2001).

33. Grieder, N.C., de Cuevas, M. \& Spradling, A.C. The fusome organizes the microtubule network during oocyte differentiation in Drosophila. Development 127, 4253-4264 (2000).

34. Theurkauf, W.E. \& Hazelrigg, T.I. In vivo analyses of cytoplasmic transport and cytoskeletal organization during Drosophila oogenesis: characterization of a multi-step anterior localization pathway. Development 125, 3655-3666 (1998).

35. Mahowald, A.P. \& Strassheim, J.M. Intercellular migration of centrioles in the germarium of Drosophila melanogaster. An electron microscopic study. J Cell Biol 45, 306-320 (1970).

36. Stevens, N.R., Raposo, A.A., Basto, R., St Johnston, D. \& Raff, J.W. From stem cell to embryo without centrioles. Curr Biol 17, 1498-1503 (2007).

37. Basto, R. et al. Flies without centrioles. Cell 125, 1375-1386 (2006).

38. Blachon, S. et al. Drosophila asterless and vertebrate Cep152 Are orthologs essential for centriole duplication. Genetics 180, 2081-2094 (2008).

39. Ni, J.Q. et al. A genome-scale shRNA resource for transgenic RNAi in Drosophila. Nat Methods 8, 405-407 (2011).

40. Hiraoka, Y. \& Dernburg, A.F. The SUN rises on meiotic chromosome dynamics. Dev Cell 17, 598-605 (2009).

41. Rothballer, A. \& Kutay, U. The diverse functional LINCs of the nuclear envelope to the cytoskeleton and chromatin. Chromosoma 122, 415-429 (2013).

42. Starr, D.A. \& Fridolfsson, H.N. Interactions between nuclei and the cytoskeleton are mediated by SUN-KASH nuclear-envelope bridges. Annu Rev Cell Dev Biol 26, 421-444 (2010).

43. Tapley, E.C. \& Starr, D.A. Connecting the nucleus to the cytoskeleton by SUN-KASH bridges across the nuclear envelope. Curr Opin Cell Biol 25, 57-62 (2013).

44. Kracklauer, M.P., Banks, S.M., Xie, X., Wu, Y. \& Fischer, J.A. Drosophila klaroid encodes a SUN domain protein required for Klarsicht localization to the nuclear envelope and nuclear migration in the eye. Fly (Austin) 1, 75-85 (2007).

45. Kracklauer, M.P. et al. The Drosophila SUN protein Spag4 cooperates with the coiled-coil protein Yuri Gagarin to maintain association of the basal body and spermatid nucleus. J Cell Sci 123, 2763-2772 (2010).

46. Technau, M. \& Roth, S. The Drosophila KASH domain proteins Msp-300 and Klarsicht and the SUN domain protein Klaroid have no essential function during oogenesis. Fly (Austin) 2, 82-91 (2008).

47. Fischer-Vize, J.A. \& Mosley, K.L. Marbles mutants: uncoupling cell determination and nuclear migration in the developing Drosophila eye. Development 120, 2609-2618 (1994). 
48. Volk, T. A new member of the spectrin superfamily may participate in the formation of embryonic muscle attachments in Drosophila. Development 116, 721-730 (1992).

49. Yu, J. et al. The KASH domain protein MSP-300 plays an essential role in nuclear anchoring during Drosophila oogenesis. Dev Biol 289, 336-345 (2006).

50. Mosley-Bishop, K.L., Li, Q., Patterson, L. \& Fischer, J.A. Molecular analysis of the klarsicht gene and its role in nuclear migration within differentiating cells of the Drosophila eye. Curr Biol 9, 1211-1220 (1999).

51. Jeffress, J.K. et al. The formation of the central element of the synaptonemal complex may occur by multiple mechanisms: the roles of the $\mathrm{N}$ - and $\mathrm{C}$-terminal domains of the Drosophila C(3)G protein in mediating synapsis and recombination. Genetics 177, 2445-2456 (2007).

52. Bowman, S.K., Neumuller, R.A., Novatchkova, M., Du, Q. \& Knoblich, J.A. The Drosophila NuMA Homolog Mud regulates spindle orientation in asymmetric cell division. Dev Cell 10, 731-742 (2006).

53. Izumi, Y., Ohta, N., Hisata, K., Raabe, T. \& Matsuzaki, F. Drosophila Pins-binding protein Mud regulates spindle-polarity coupling and centrosome organization. Nat Cell Biol 8, 586593 (2006).

54. Merdes, A., Ramyar, K., Vechio, J.D. \& Cleveland, D.W. A complex of NuMA and cytoplasmic dynein is essential for mitotic spindle assembly. Cell 87, 447-458 (1996).

55. Nguyen-Ngoc, T., Afshar, K. \& Gonczy, P. Coupling of cortical dynein and G alpha proteins mediates spindle positioning in Caenorhabditis elegans. Nat Cell Biol 9, 1294-1302 (2007).

56. Siller, K.H., Cabernard, C. \& Doe, C.Q. The NuMA-related Mud protein binds Pins and regulates spindle orientation in Drosophila neuroblasts. Nat Cell Biol 8, 594-600 (2006).

57. Yu, J.X., Guan, Z. \& Nash, H.A. The mushroom body defect gene product is an essential component of the meiosis II spindle apparatus in Drosophila oocytes. Genetics 173, 243-253 (2006).

58. Page, S.L. \& Hawley, R.S. c(3)G encodes a Drosophila synaptonemal complex protein. Genes Dev 15, 3130-3143 (2001).

59. Page, S.L. et al. Corona is required for higher-order assembly of transverse filaments into fulllength synaptonemal complex in Drosophila oocytes. PLoS Genet 4, e1000194 (2008).

60. Paddock, S.W. \& Albrecht-Buehler, G. The degree of coupling of nuclear rotation in binucleate 3T3 cells. Exp Cell Res 166, 113-126 (1986).

61. Paddock, S.W. \& Albrecht-Buehler, G. Distribution of microfilament bundles during rotation of the nucleus in 3T3 cells treated with monensin. Exp Cell Res 163, 525-538 (1986).

62. Pomerat, C.M. Rotating nuclei in tissue cultures of adult human nasal mucosa. Exp Cell Res 5, 191-196 (1953).

63. Szikora, S., Gaspar, I. \& Szabad, J. 'Poking' microtubules bring about nuclear wriggling to position nuclei. J Cell Sci 126, 254-262 (2013).

64. Koszul, R. \& Kleckner, N. Dynamic chromosome movements during meiosis: a way to eliminate unwanted connections? Trends Cell Biol 19, 716-724 (2009).

65. Woglar, A. \& Jantsch, V. Chromosome movement in meiosis I prophase of Caenorhabditis elegans. Chromosoma 123, 15-24 (2014).

66. Sanchez, T., Chen, D.T., DeCamp, S.J., Heymann, M. \& Dogic, Z. Spontaneous motion in hierarchically assembled active matter. Nature 491, 431-434 (2012).

67. Zhao, T., Graham, O.S., Raposo, A. \& St Johnston, D. Growing microtubules push the oocyte nucleus to polarize the Drosophila dorsal-ventral axis. Science 336, 999-1003 (2012).

68. Cain, N.E., Tapley, E.C., McDonald, K.L., Cain, B.M. \& Starr, D.A. The SUN protein UNC84 is required only in force-bearing cells to maintain nuclear envelope architecture. J Cell Biol 206, 163-172 (2014).

69. Jauffred, B. et al. Regulation of centrosome movements by numb and the collapsin response mediator protein during Drosophila sensory progenitor asymmetric division. Development 140, 2657-2668 (2013).

70. Gepner, J. et al. Cytoplasmic dynein function is essential in Drosophila melanogaster. Genetics 142, 865-878 (1996). 


\section{FIGURE LEGENDS}

\section{Figure 1: Centromeres and nuclei of 8-cell cysts display a dynamic rotation behavior}

(A) germline stem cells (GSCs) at the anterior tip of the germarium (left) produce cystoblasts (CB). Spectrosome (red circles) in GSCs and CBs, which develops into a branched fusome. In early region $2 \mathrm{a}$, the synaptonemal complex (red lines) forms along the chromosomes of the two cells with four ring canals (pro-oocytes, yellow) as they enter meiosis. By region $2 \mathrm{~b}$, the oocyte (Oo) is selected and is the only cell to remain in meiosis. Terminal Filament (TF), Cap cells (Cap), Follicle Cells (FC). Blue dots are centrosomes migrating into the oocyte. Green shade shows the progressive restriction of some proteins or mRNAs into the oocyte. From Christophorou et $\mathrm{al}^{23}$.

(B) Projection of $11 \mathrm{Z}$-sections of a living germarium expressing CID::RFP (centromere, red) and Par1::GFP (fusome, green). GSC (arrowhead), CB (arrow) and an 8-cell cyst (8cc), whose cells are linked by a fusome. Four nuclei of the $8 \mathrm{cc}$, are surrounded by dotted lines. $(\mathrm{C}, \mathrm{D})$ Selected projections from boxed regions in B showing a single $\mathrm{CB}$ nucleus $(\mathrm{C})$ and a single 8cc nucleus (D) over a 3-mn time course (nuclear surfaces are indicated by a dotted circle in each image). Time-colored trackings for three CID-RFP dots (arrows) in C and for one CID-RFP dot (arrowhead) in D are shown in right panels.

(E, F) 3D representations indicating the covered volume of one selected representative track for all time points, the ellipsoid is arbitrarily centered into a sphere representing the nuclear volume of a cystoblast (E) or an 8cc (F).

(G) Raw covered volume plots for each track according to cyst stage. For each track, the total covered volume and the track duration is indicated along the $\mathrm{y}$ and $\mathrm{x}$ axes, respectively.

(H) Distribution of the relative covered volume (raw covered volume/ nuclear volume) per second for each track at different cyst stages (S.D., Mann-Whitney U-test comparing wildtype $8 \mathrm{cc}$ with other stages, $\mathrm{p} \leq 1 \times 10^{-4}, \mathrm{n}=6$ experiments).

(I) Selected projections showing a single $8 \mathrm{cc}$ nucleus over a $3 \mathrm{~min}$. time course A UV pulse photoconverts Dendra2 from a green to red emitting protein. Time-colored tracking for one CID-RFP dot (red arrowhead) and one activated H2-dendra dot (red arrow) is shown at the end of the time-lapse sequence. Time in minutes and scale bars represent $2 \mu \mathrm{m}$.

\section{Figure 2: Microtubules drive centromere movements.}


(A) Z-projection of CID::RFP (centromeres, red), asl::YFP (centrosomes, yellow) and Jupiter::GFP (microtubules, green). Microtubules emanate from the fusome (empty arrowhead), nuclear membrane (arrow) and centrosomes (filled arrowhead).

(B) Z-projection CID::RFP (centromeres, red), asl::YFP (centrosomes, yellow) and Jupiter::GFP (microtubules, green). Immediately after a $5 \mathrm{~s}$ UV pulse, microtubules re-grow from the fusome (empty arrowheads), centrosomes (filled arrowheads) and nuclear membrane (arrow).

(C) $35 \%$ of centrosomes display rotations, while $65 \%$ remained static.

(D) $87 \%$ of centrosomes were rotating in the same direction and with the same speed as centromeres of the same cell.

(E) During these co-rotations, centromeres and centrosomes co-localized at the nuclear envelop in only $28 \%$ of cases examined.

(F) In static nuclei, centromeres and centrosomes co-localized and were associated with the fusome in $91 \%$ of cases examined.

(G) Selected projections showing a single 8cc nucleus over a 1.5-mn time course. Centrosomes (asl::YFP, green) and centromeres (CID::RFP, red). Centrosomes rotate in the same direction and with the same speed as centromeres. Colored tracking for one CID-RFP dot (red arrowhead) and one centrosome dot (green arrowhead) is shown on right panel.

(H) Selected Z-projection of a single 8cc nucleus expressing CID::RFP over a 3-mn time course (nuclear surface is indicated by a dotted circle in each image). Right panel: timecolored tracking (arrow).

(I) Inactivation of colcemid leads to reestablishment of CID foci dynamics in $8 \mathrm{cc}$. Selected Z-projections of a single 8cc nucleus are shown. In the first two projections colcemid is active, microtubules are depolymerized and centromeric foci movement is very limited. In the last three projections colcemid was inactivated and centromeric movement is gradually restored. For each time point, the cumulative tracking is represented in the bottom half picture. The yellow and white dotted circles indicate the nuclear surface of two nuclei in each image.

$(\mathrm{J}, \mathrm{J}$ ') 3D representations indicating the covered volume of one representative track (yellow nucleus), the ellipsoid is arbitrarily centered into a sphere representing the nuclear volume of an $8 \mathrm{cc}$ nucleus before the UV pulse (J) and the same $8 \mathrm{cc}$ after UV pulse (J').

(K) The relative covered volume (raw covered volume/ nuclear volume) per second in $8 \mathrm{cc}$ nuclei treated with colcemid is strongly reduced compared to wild-type 8cc (S.D., MannWhitney U-test, $\mathrm{p} \leq 1 \times 10^{-4} ; \mathrm{n}=7$ experiments). 
Figure 3: sas-4, asl and Dynein loss of function affects centromere dynamics.

(A-D) Loss of function of Sas-4 and Asl (centrosomes) and Dynein by RNAi leads to inhibition of CID foci dynamics in living 8 cell-cysts. Selected projections of Z-sections obtained by time lapse microscopy (spinning disc) of CID-RFP; nos $>w$-shRNA (A) CIDRFP/sas-4-shRNA;nos-Gal4/+ (B), CID-RFP/asl-shRNA; nos-Gal4/+ (C) and CID$R F P / d h c 64 c-s h R N A^{36583}$; nos-Gal4/+ (D) germaria displaying a single 8cc nucleus over a 3mn time (nuclear surface is indicated by a dotted circle in each image) are shown. Timecolored trackings of one or two CID-RFP dots (arrowhead or arrows) are shown at the end of the time-lapse sequence.

(E-H) 3D representations indicating the covered volume of one selected representative track for all time points of a CID-RFP; nos $>w$-shRNA nucleus (E), a CID-RFP/sas-4shRNA;nos-Gal4/+ (F), a CID-RFP/asl; nos-Gal4/+ (G) and a CID-RFP /Dhc64c-shRNA ${ }^{36583}$ IR; nos-Gal4/+ nucleus $(\mathrm{H})$. The ellipsoid is arbitrarily centered into a sphere representing the nuclear volume (gold sphere).

(I) Distribution of the relative covered volume (raw covered volume/ nuclear volume) per second of centromeres in wild type, sh-RNAs for sas-4, asl and $d h c 64 c$, and $D h c 64 C^{3-2}$ $/ D h c 64 C^{6-12}$ mutant germaria (error bars are SD; Mann-Whitney U-test $\mathrm{p} \leq 1 \times 10^{-4}$; nos $>\mathrm{W}-$ shRNA $n=44 / 6$ experiments, nos $>$ sas-4-shRNA $n=37 / 5$ experiments, nos $>$ asl-shRNA $n=80 / 5$ experiments, nos $>$ Dhc-shRNA36583 $n=55 / 5$ experiments, nos $>$ Dhc-shRNA36698 $n=55 / 5$ experiments, Dhc- $64^{6-12} / D h c-64^{3-2} n=24 / 3$ experiments).

Figure 4: Microtubules, centrosomes and Dynein are required for centromere pairing in $8 \mathrm{cc}$ and assembly of the synaptonemal complex.

(A, E) Projection of Z-sections obtained by DV microscopy of wild-type (A), colcemid treated (B), sas-4 ${ }^{S 2214}$ (C), asl ${ }^{\mathrm{mecD}}$ (D) and nos-Gal4>Dhc64C-shRNA ${ }^{36698}$ (E) fixed germaria stained for centromeres (CID, red), and DNA. 8-cell cyst nuclei are indicated by a dotted circle in each image. Scale bars represent $2 \mu \mathrm{m}$.

(F) Developmental changes in the number of CID foci in 8cc nuclei and in pachytene nuclei in region $2 \mathrm{a}$ in wild-type, colcemid treated and in different mutant and RNAi conditions. The number of analyzed cells is indicated under each stage on the right panel, (3 independent experiments for each genotype; error bars are S.D.; two-tailed Student's t-test, ${ }^{*} \mathrm{p} \leq 5 \times 10^{-}$ $\left.2, * * \mathrm{p} \leq 5 \times 10^{-5}, * * * \mathrm{p} \leq 5 \times 10^{-8}\right)$ 
(G-K") Projection of Z-sections obtained by DV microscopy of a wild-type (G-G”), colcemid treated (H-H”), sas-4 (I-I"), asl ${ }^{\text {mecD }}$ (J-J") and nos-Gal4>Dhc64C-shRNA ${ }^{36698}$ (K-K”) fixed germaria stained for centromeres $(\mathrm{CID}, \mathrm{red})$ and the synaptonemal complex $(\mathrm{C}(3) \mathrm{G}$, green). Scale bars represent $2 \mu \mathrm{m}$.

(L) Changes in the length of the SC in colcemid treated and in loss of function of Dynein and centrosomes compared to wt ( $\mathrm{Y}$ axis is in $\mu \mathrm{m} ; 5$ independent nuclei for each genotype; error bars are S.D.; two-tailed Student's t-test, $\mathrm{p}<0.01$ ).

Figure 5: Klarsicht and Klaroid are present near centromeres in the mitotic region and are differentially required for chromosome movements.

(Aa-Ad) Projection of Z-sections obtained by DV microscopy of a wild-type 8cc stained for centromere (CID, orange), Klarsicht (Klar, green), Klaroid (Koi, magenta) and DNA (DAPI, blue). Scale bar represents $2 \mu \mathrm{m}$.

(B) Selected projections of $5 \mathrm{Z}$-sections obtained by time lapse microscopy (confocal) of a living CID-RFP; nos>UAS-KASH-GFP germarium showing a single 8cc nucleus over a 3 min time course. Colored tracking for one CID-RFP dot (arrowhead, red) and one KASHGFP dot (arrowhead, green) is shown at the end of the time-lapse sequence.

(C, D) Selected projections of Z-sections obtained by time lapse microscopy (spinning disc) of $C I D:: R F P /+; k l a r^{m b C D 4}(\mathrm{C})$ and $C I D:: R F P, k^{20} i^{80} / k^{\circ i} i^{80}$ (D) germaria displaying single $8 \mathrm{cc}$ nuclei over a 3-mn time course (nuclear surface is indicated by a dotted circle in each image) are shown. Time-colored trackings of CID-RFP dots (arrowhead or arrow) are shown at the end of the time-lapse sequence.

(E,F) 3D representations indicating the relative covered volume of one selected representative track for all time points of a CID::RFP/+;klar ${ }^{m b C D 4}(\mathrm{E})$, and a $C I D:: R F P, k o i^{80} / k^{2} i^{80}(\mathrm{~F}) 8 \mathrm{cc}$ selected nucleus. The ellipsoid is arbitrarily centered into a sphere representing the nuclear volume (gold sphere).

(G) Distributions of the relative covered volume per second for centromeric foci in CID-RFP, $C I D:: R F P /+$;klar ${ }^{m b C D 4}$, and CID::RFP, $k o i^{80} / \mathrm{koi}^{80} 8 \mathrm{cc}$ nuclei (error bars are S.D., MannWhitney U-test $\mathrm{p} \leq 1 \times 10^{-3} ; \mathrm{n}=12$ experiments).

Figure 6: klarsicht and klaroid loss of function affect centromeric pairing in 8cc and synaptonemal complex assembly in pachytene nuclei.

(A-C) Projection of Z-sections obtained by DV microscopy of $k l a r^{\text {marb-CD4 }}$ (A), koi ${ }^{80}$ (B) and $k i^{80} ; k^{2} r^{\text {marb-CD4 }}(\mathrm{C})$ fixed germaria stained for centromere (CID, red), fusome ( $\alpha$-spectrin, 
green), and DNA (DAPI, blue). Nuclei of 8-cell cysts are indicated by a dotted circle. Scale bars represent $2 \mu \mathrm{m}$.

(D) Developmental changes in the number of CID foci in 8cc nuclei and in pachytene nuclei in region 2a in wild-type, $k_{l a r}{ }^{\text {marb-CD4 }}$, nos $>$ klar_shRNA, $k o i^{80}$, nos $>k o i \_s h R N A$ and $k^{2} i^{80}$; $k{ }^{\text {marb-CD4 }}$ fixed germaria. The number of analyzed cells is indicated under each stage (3 independent experiments for each genotype; error bars are S.D.; two-tailed Student's t-tests * $\left.\mathrm{p} \leq 5 \times 10^{-2}, * * \mathrm{p} \leq 5 \times 10^{-5}, * * * \mathrm{p} \leq 5 \times 10^{-8}\right)$.

(E-H') Projection of Z-sections obtained by confocal microscopy of a wild-type (E, E'),

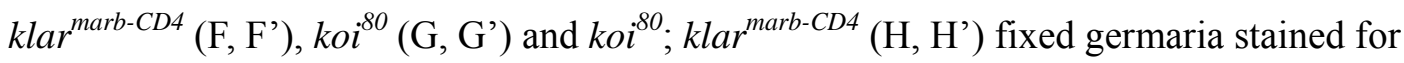
synaptonemal complex $(\mathrm{C}(3) \mathrm{G}$, red). E, F, G and H correspond to 16-color conversions of projections E', F', G' and H' respectively to better illustrate signal intensity differences (ImageJ: Image: Lookup tables: 16 colors). Germaria are indicated by a dotted line. Scale bars represent $20 \mu \mathrm{m}$.

(I) Changes in the percentage of germaria displaying SC defects in wild-type, klar $^{\text {marb-CD4 }}$,

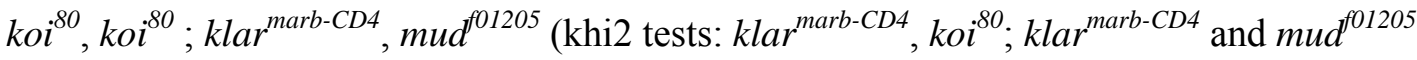
$\left.\mathrm{p} \leq 5 \times 10^{-5}\right)$. The number of analyzed germaria is indicated under each stage. 3 independent experiments of each genotype.

(J) SC fluorescence intensity was quantified in sas-4 $4^{S 2214}, a s l^{\mathrm{MecD}}, \mathrm{klar}^{\text {marb-CD4 }}, \mathrm{koi}^{80}$ and

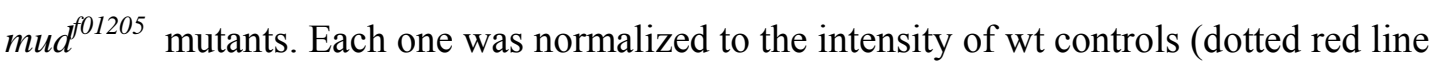
equal to 1) introduced in the mutant preparation (3 experiments; two-tailed Student's t-tests * $\left.\mathrm{p} \leq 5 \times 10^{-2}, * * \mathrm{p} \leq 5 \times 10^{-5}, * * * \mathrm{p} \leq 5 \times 10^{-8}\right)$.

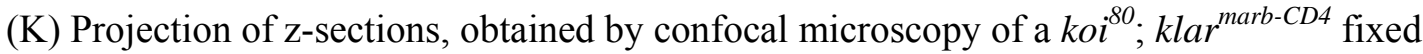
germarium stained for synaptonemal complex (C(3)G, red), and DNA (DAPI, blue). Scale bar represents $20 \mu \mathrm{m}$.

(L) A polycomplex from the corresponding boxed region in $\mathrm{J}$ is shown. Scale bar represents 1 $\mu \mathrm{m}$.

Figure 7: Mud associates with Klarsicht and Klaroid in 8 cell-cysts close to centromeres, but is not required for chromosome movements.

(A) Projection of Z-sections obtained by confocal microscopy of a wild-type fixed germarium stained for MUD (green) and SC (C(3)G, red). Scale bar represents $10 \mu \mathrm{m}$.

$(\mathrm{B}, \mathrm{C}) \mathrm{B}$ is a projection of Z-sections, obtained by confocal microscopy of a wild-type fixed germarium stained for MUD (green), the centromeres (CID, red) and the nuclear membrane 
(WGA, blue). A close up on a CID-MUD association (C) from the corresponding boxed region in B is shown. Scale bars represent $2 \mu \mathrm{m}$ and $0.5 \mu \mathrm{m}$ in B and C respectively.

(Da-Dd) Projection of Z-sections obtained by DV microscopy of a wild-type 8cc stained for centromeres (CID, orange), Mud (Mud, green), Klarsicht (Klar, magenta) and DNA (DAPI, blue).

(Ea-Ed) Projection of Z-sections obtained by DV microscopy of a wild-type 8cc stained for centromeres (CID, orange), Mud (Mud, green), Klaroid (Koi, magenta) and DNA (DAPI, blue).

$(\mathrm{F}, \mathrm{G}) \mathrm{mud}^{\mathrm{f01205}}$ mutation has little effect on CID foci dynamics in 8-cell cysts. Selected projections of a CID-RFP (F) and $m u d^{f 01205}$; CID-RFP (G) germarium showing a single 8cc nucleus over a 3-min time course (nuclear surface is indicated by a dotted circle in each image). The corresponding time-colored tracking for one CID-RFP dot (arrowhead) is shown at the end of each time-lapse sequence.

(H, I) 3D representations indicating the relative covered volume of one selected track for all time points of a CID-RFP (H) and a mud ${ }^{f 01205}$; CID-RFP (I) 8cc nucleus. The ellipsoid is arbitrarily centered into a sphere representing the nuclear volume (gold sphere) of an $8 \mathrm{cc}$ stage.

(J) Distributions of the relative covered volume per second for centromeric foci in CID-RFP and $m u d^{f 01205}$; CID-RFP 8cc nuclei. Mann-Whitney U-test comparing CID-RFP with mud $^{\text {f01205 }}$; CID-RFP 8cc nuclei ( $\left.\mathrm{p}=0.37\right)$.

Figure 8: mud plays a minor role in centromere pairing in 8cc but is required to maintain the nuclear envelop integrity and for the assembly of the synaptonemal complex.

(A, B) Z-projections of $k o i^{80} /+(\mathrm{A})$ and $m u d^{f 01205}$ (B) fixed germaria stained for centromeres (CID, red), fusome ( $\alpha$-spectrin, green), and DNA (DAPI, blue). Nuclei of an 8cc with a branched fusome are shown. Scale bars represent $2 \mu \mathrm{m}$.

(C) Developmental changes in the number of CID foci for 8cc cell stage and in pachytene nuclei in region 2a in wild-type and different mutant and sh_RNA conditions. The number of analyzed cells is indicated under each stage (3 independent experiments for each genotype; error bars are S.D.; two-tailed Student's t-tests: $* \mathrm{p} \leq 5 \times 10^{-2}, * * \mathrm{p} \leq 5 \times 10^{-5}, * * * \mathrm{p} \leq 5 \times 10^{-8}$; twotailed Student's t-tests with Bonferonni correction after ANOVA: $+\mathrm{p} \leq 1,67 \times 10^{-2},++$ $\left.\mathrm{p} \leq 1,67 \times 10^{-5},+++\mathrm{p} \leq 1,67 \times 10^{-8}\right)$ 
(D-E') Z-projections of a wild-type (D, D') and $m u d^{f 01205}$ (E, E') fixed germaria stained for $\mathrm{SC}(\mathrm{C}(3) \mathrm{G}$, red). D and E correspond to 16-color conversions of projections D' and E' respectively to better illustrate signal intensity differences. Scale bars represent $20 \mu \mathrm{m}$.

(F) Z-projections of wild stage 3 egg chamber stained for MUD in green, C(3)G in red and DNA.

$(\mathrm{G}, \mathrm{H}) \mathrm{Z}$-projections of wild type $(\mathrm{G})$ and $m u d^{f 01205}(\mathrm{H})$ stage 3 egg chambers stained for $\mathrm{C}(3) \mathrm{G}$ in red, the nuclear membrane (lamin, green), and DNA. When PCs are observed in $m u d^{f 01205}$ DNA in the corresponding oocyte is diffuse and the nuclear membrane has collapsed.

(I) Z-projections of wild type stage 3 egg chamber stained for $\mathrm{C}(3) \mathrm{G}$ in red, Orb in green and DNA. Oocytes with PCs in $m u d^{f 01205}$ mutants still display polarized localization of Orb.

(J-K') Z-projections obtained by structured illumination microscopy of C-term C(3)G (green) and Cona (red) on pachytene nuclei from wild type and polycomplexes from $m u d^{f 01205}$. (J) In wild type pachytene nuclei Cona localizes between the two threads of C(3)G. (J') Line profiles plot the normalized intensity for Cona (red) and C(3)G (green) from panel (J). The Cona peak is seen between the two parallel peaks of C(3)G. (K) Structured illumination microscopy of polycomplexes in $m u d^{f 01205}$ germaria showed there is still an alternate although disorganized structure where Cona localises between the two threads of C(3)G. (K') Line profiles from panel (K) confirm that in PCs Cona still localizes between the two threads of $\mathrm{C}(3) \mathrm{G}$. 


\section{MATERIALS AND METHODS}

\section{Fly stocks and genetics}

For all experiments on fixed and live germaria the following strains were used: $w^{1118}$ was used as the wild-type strain when assaying centromere pairing and clustering and $y^{1} w^{1118} \mathrm{hs}-\mathrm{Flp}$; FRT82B::GFP as the wild type strain when assaying synapsis. For testing mutants, the following strains were used: $w^{*} ;$ sas $-4^{\text {s2214 37 }}$, asl $^{\text {mecD }}{ }^{38}$, koi $^{\text {HRKO80.w 51, 58 }}$, klar $^{\text {mCD4 59 }}, w^{1118}$, $m u d^{f 0120569}, D h c 64 c^{3-2} / D h c 64 c^{6-1270}$. For sh-RNAs the following lines were used: UASp::Trip white (Bloomington: 35573); UASp::Trip Dhc64c (Bloomington: 36698 and 36583);

UASp::Trip sas-4 (Bloomington:35049); UASp::Trip asl (Bloomington: 38220); UASp::Trip klar (Bloomington: 36721); UASp::Trip koi (Bloomington: 40924); UASp::Trip mud (Bloomington: 38190 and 35044) (Ni et al., 2011). Also, for experiments on live germaria CID::RFP/+ ${ }^{29}$; nos-Gal4/UASp::Par1:GFP/+ (fusome marker gift from Daniel St Johnston); $\left(\mathrm{w}\left[{ }^{*}\right], \mathrm{P}\{\mathrm{w}[+\mathrm{mC}]=\right.$ GFP-Nup107.K $\} 13.2 .1 ; \mathrm{wg}[\mathrm{Sp}-1] / \mathrm{CyO}$ (Bloomington: 35513); pUASp-GFP:KASH/CyO ${ }^{46} ; \mathrm{w}[1118] ; \mathrm{P}\{\mathrm{w}[+\mathrm{mC}]=\mathrm{PTT}-\mathrm{GA}\}$ Jupiter[G00147] (Bloomington: 6836) and P\{Ubi-YFP-asl.FL $\}$ were used.

\section{Immunohistochemistry}

For immunostaining, ovaries were dissected in PBS, fixed in 4\%PFA-PEPS, permeabilized in PBT (0,2\% Triton) for 30 min, left overnight with primary antibodies in PBS at $4{ }^{\circ} \mathrm{C}$, washed 4 x 30 min in PBS, left with secondary antibody for $2 \mathrm{hr}$ at room temperature, washed 4 x 30 min in PBS where DAPI (1:500) was added during the last wash and mounted in Cityfluor. We used the following primary antibodies: mouse anti-C(3)G 1A8-1G2 (1:500), rabbit antiC(3)G (1:1000) and guinea pig anti-Cona (1:500) (gifts from Scott Hawley, Stowers Institute, USA), rat anti-Cid (1:1000) (gift from Claudio E. Sunkel, Universidade do Porto, Portugal), 
rabbit anti- $\alpha$ Spectrin (1:1000) (gift from R. Dubreuil) to label the fusome and identify the cyst stages, rabbit anti-Mud $(1: 500)^{57}$, rat anti-Klaroid (1:200), guinea pig anti-Klarsicht (1:200) (gifts from Michael Welte and Janice Fischer); mouse anti-Orb (1:500) (DSHB); mouse anti-Lamin (1:500) (ADL84.12, DSHB). Secondary antibodies conjugated with Cy3, Cy5 and FITC (Jackson laboratories) were used at 1/200. For lectin staining WGA conjugated to alexa 488 was used (1:500).

\section{Colcemid treatments}

Colcemid was added to the fly medium at a concentration of $0,2 \mathrm{mg} / \mathrm{mL}$ diluted in saccharose $1 \%$ and added to dry yeast. To assay pairing and clustering of centromeres, long-term drug treatments lasted $48 \mathrm{~h}$. Freshly prepared drug was added every $12 \mathrm{~h}$. For live imaging, adult flies were fed for four hours with the colcemid-containg food. Ovaries were dissected as above and live imaging was performed as described below. Colcemid was inactivated with a brief UV pulse (5sec) using an Inverted Spinning disk Confocal Microscope (Roper/Nikon). Flies expressing CID:RFP and Jupiter:GFP to see the microtubules were used as control flies to confirm the colcemid inactivation after the UV pulse.

\section{Image acquisition and data analysis}

DV Images of fixed germaria were collected under a DeltaVision deconvolution microscope system (Applied Precision) equipped with an Olympus 1670 inverted microscope and CoolSNAP HQ camera (Photometrics). All images were acquired with the PlanApo 60x/1,42 oil objective lens with $1.5 \mathrm{X}$ auxiliary magnification at $0.2 \mu \mathrm{m}$ intervals along $\mathrm{z}$-axis and deconvolved using the softWoRx v.3.5.1 software (Applied Precision). Confocal Images of fixed germaria were collected under a Zeiss LSM 700 NLO confocal. All images were acquired with a PlanApo 63x/1,40 oil objective at 0.6 um intervals along z-axis and operated by ZEN 2012 software. 
When structured illumination was performed we used a rotary stage OMX v3 system (Applied Precision- GE healthcare), equipped with 3 EMCCD, evolve cameras (photometrics). Signal from all channels were realigned using fluorescent beads prior to each session of image acquisition. Registration was done using UnwarpJ in ImageJ (Rasband, WS). All images were acquired with a PlanApo 100x/1,4 oil objective at $125 \mathrm{~nm}$ intervals along z-axis. Pixel size is $40 \mathrm{~nm}$ along $\mathrm{xy}$-axis after reconstruction.

The length of the synaptonemal complex was measured from DV images with a macro on ImageJ designed by Olivier Leroy from the BDD imaging facility.

Fluorescence quantification and normalization was performed on confocal images. In brief, from each slide preparation pictures of $\mathrm{C}(3) \mathrm{G}$ staining of mutant or sh-RNA germaria and control germaria (GFP+) were taken under exactly the same settings (exposure, image size, bit depth, acquisition speed, etc...). Fluorescence emitted per germarium was quantified and a mean \pm st.dev was calculated. Finally the mean fluorescence of mutant germaria was normalized to the mean of fluorescence of control germaria. In the case of sas-4 clones, fluorescence from individual nuclei was quantified.

For live imaging ovaries were dissected in oil (10S, Voltalef, VWR). The muscular sheath around each ovariole was removed and germaria were made to stick to coverslips in oil. Movies were collected with an Inverted Spinning disk Confocal Microscope (Roper/Nikon) operated by Metamorph on an inverted Nikon Eclipse Ti microscope coupled to an Evolve EMCCD camera (Photometrics) or a CoolSNAP HQ2 camera (Photometrics) for Colcemid experiments and temperature control chamber. All images were acquired with the Planapo $60 x / 1,4$ oil objective lens with $1.5 \mathrm{X}$ auxiliary magnification. Single position movies in the germarium were acquired for $8 \mathrm{~min}$ at $25 \pm 1{ }^{\circ} \mathrm{C}$, with a $10 \mathrm{sec}$ temporal resolution ( 11 slices $Z$ stack, $0.5 \mu \mathrm{m} / \mathrm{slice})$. For colcemid experiments, single position movies in the germarium were 
acquired for $30-40 \mathrm{~min}$ at $25 \pm 1^{\circ} \mathrm{C}$, with a $30 \mathrm{sec}$ temporal resolution ( 7 slices $Z$-stack, 0.7 $\mu \mathrm{m} / \mathrm{slice})$.

The use of $\alpha$-spectrin and Par1 on fixed and live germaria respectively allowed the identification of the different cyst stages. The quantification of CID foci on fixed germaria, was performed like previously described (Christophorou et al., 2013). In all figures the images of fixed germaria shown are the projection of all z-series which cover a region ranging from the first CID foci until the last CID foci seen. For live germaria images shown are the projection of all z-series of a single ( $\mathrm{t}$ ) projection.

3D tracking of spinning disk data was performed using Imaris software (Bitplane). The CID::RFP signal was tracked using the "spots" function with an expected diameter of $0.3 \mu \mathrm{m}$. Automatically generated tracks were then edited manually in order to eliminate inappropriate connections, including connections between foci in different nuclei or between foci of different sizes or intensity when more likely assignments were apparent or multiple spots assigned to the same focus.

To remove global movements of the germarium, each nucleus containing a CID::RFP focus was assigned to the nearest fusome foci. Then, position of the reference fusome was substracted to each CID::RFP foci for each time point of the tracking in order to get the relative tracks. These relatives tracks were then compiled using a custom MATLAB (MathWorks) routine that computes the minimum volume of the ellipsoid that encloses all the $3 \mathrm{D}$ points of the trajectory.

\section{Analysis of centromere trajectories}

Positions of individual centromere were tracked every 10 s during $8 \mathrm{~min}$ in order to quantify the volume covered by each centromere. This raw volume was then corrected both for overall movements of the tissue and for variations in total nuclear volume. First, we subtracted the 
motion of the germarium using the position of the fusome as a reference within each cyst (Figure 1G). Second, to take into account the significant decrease of the nuclear volume from GSCs to 16-cell cysts (Supplementary Fig. 1A), we computed the relative volume which is the raw volume divided by the mean value of the nuclear volume at each stage. Finally, we normalized durations of each track by calculating the relative covered volume per second (Figure 1H).

Analysis of correlations between tracks was done using Matlab (Mathworks, http://www.mathworks.com/ ) (Supplementary Fig. 1B). To make the analysis statistically significant, we considered only pairs of tracks $\left(\overrightarrow{r_{1}}, \overrightarrow{r_{2}}\right)$ within the same nuclei having at least 15 common time-points. For all pairs, we first computed the correlation coefficient

$$
R\left(\overrightarrow{r_{1}}, \overrightarrow{r_{2}}\right)=\frac{\left\langle\left(\overrightarrow{r_{1}}-\left\langle\overrightarrow{r_{1}}\right\rangle\right)\left(\overrightarrow{r_{2}}-\left\langle\overrightarrow{r_{2}}\right\rangle\right)\right\rangle}{\sqrt{\left\langle\left(\overrightarrow{r_{1}}-\left\langle\overrightarrow{r_{1}}\right\rangle\right)^{2}\right\rangle} \sqrt{\left\langle\left(\overrightarrow{r_{2}}-\left\langle\overrightarrow{r_{2}}\right\rangle\right)^{2}\right\rangle}}
$$

$R$ is equal to +1 when $\overrightarrow{r_{1}}$ and $\overrightarrow{r_{2}}$ are perfectly correlated, 0 when they are not and -1 when they are perfectly anti-correlated. One needs to distinguish between situations where the two tracks are actually correlated ( $R$ close to 1$)$ and situations where they are not, while centroids move in a similar / identical fashion (one compelling example would be two centroids moving in the same wise along two symmetrical arcs of circle: the two tracks are similar but $R$ is not close to 1). For the two stages SC and 8cc, the number of pairs of tracks exhibiting strong correlation $(R \geq 0.95)$ were identical (4.9\% of the total amount of long-enough pairs of tracks for $8 \mathrm{cc}$ stage) and (5.8\% for SC stage). To account for similarity for the remaining pairs of tracks that are not correlated, we built a coefficient $g$ based on the distance $D=\sqrt{\vec{r}^{2}}=\sqrt{\left(\overrightarrow{r_{1}}-\overrightarrow{r_{2}}\right)^{2}}$ between the two tracks: $g=1-\left\langle\frac{(\vec{r} \cdot \vec{v})^{2}}{\|\vec{r}\|^{2}\|\vec{v}\|^{2}}\right\rangle$ where $\vec{v}=\overrightarrow{v_{1}}-\overrightarrow{v_{2}}\left(\overrightarrow{v_{1,2}}\right.$ being the difference vector of $\left.\overrightarrow{r_{1,2}}\right),\|\quad\|$ is the norm of the vector, and $\langle\quad\rangle$ denotes the mean. $g$ gives a value between 0 and +1 inclusive since $g=1-\left\langle\cos ^{2}(\vec{r}, \vec{v})\right\rangle$. If D is constant: $g=1$ and the two 
tracks are tied / linked / bound (like two points belonging to a rigid body - solid motion) whereas $g \sim 0.5$ stands for a random $D$. According to simulations, we can consider that the two tracks are moving in an homogeneous/ related way when $>0.75$. 
A

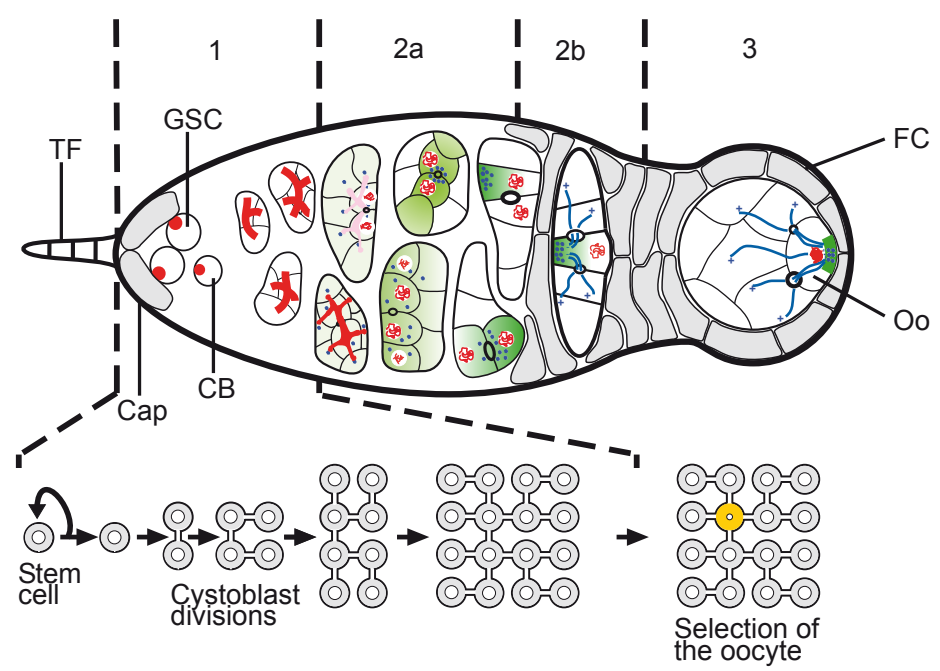

B

um
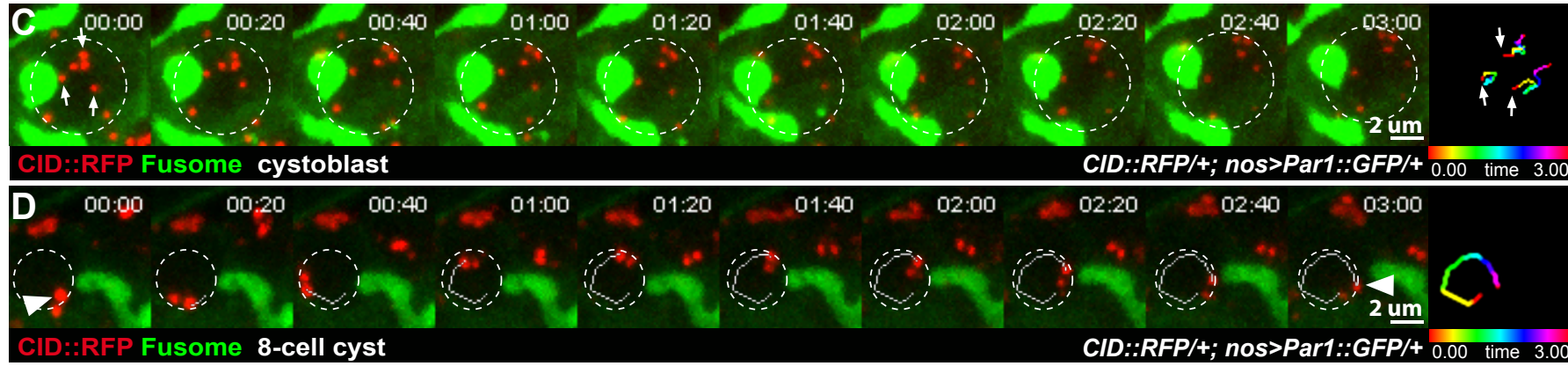

E
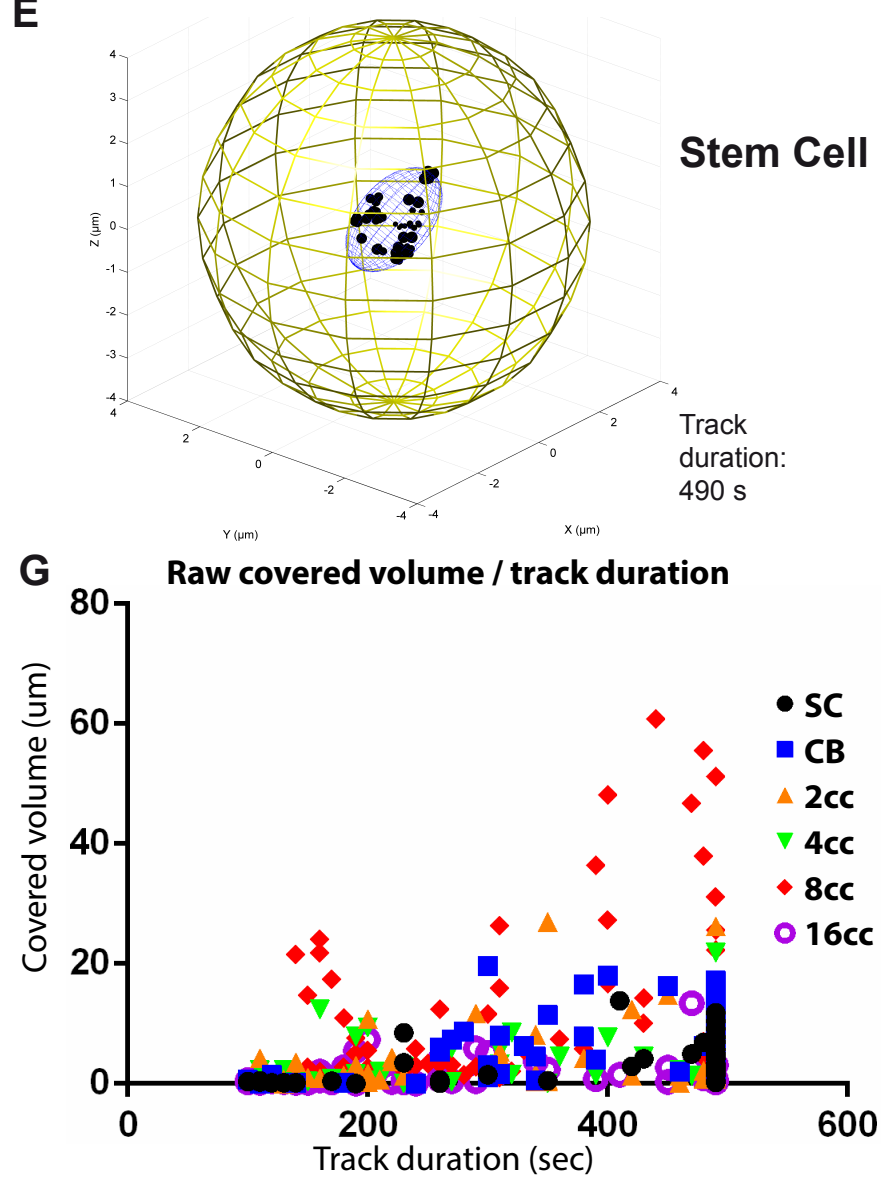

$\mathbf{F}$

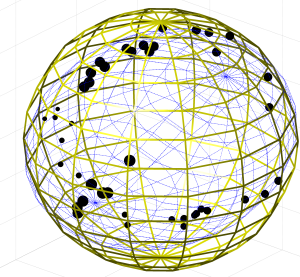

8cc

Track

duration: $440 \mathrm{~s}$
H Relative covered volume / sec

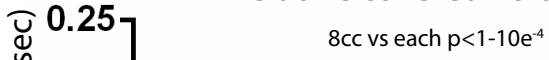

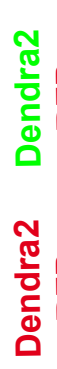

品

I $00: 00$
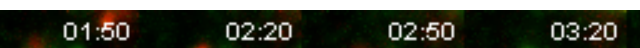

$\stackrel{n}{\varrho} 0.20-$

害 0.15 -

웡 0.10

ठั้ 0.05

$\sum_{\frac{\pi}{0}}^{0} 0.00-20$ ?

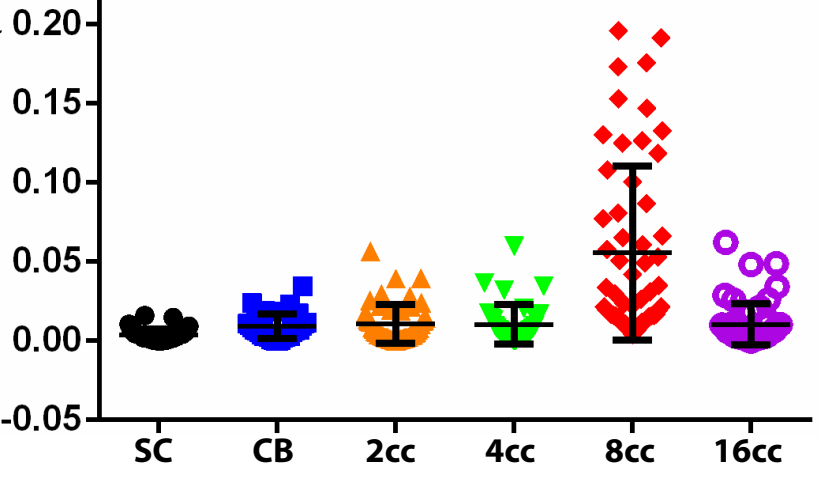

stage 

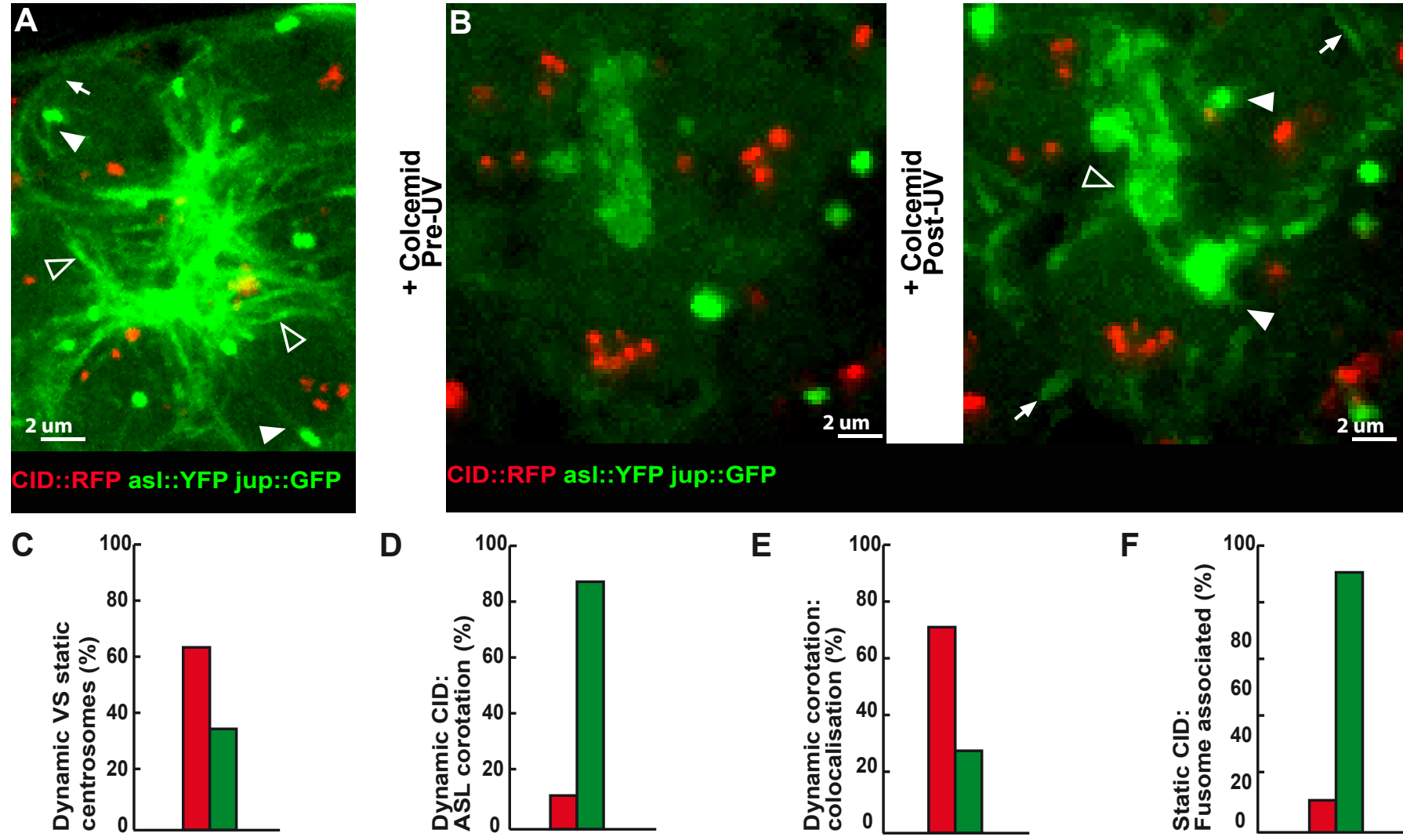

D

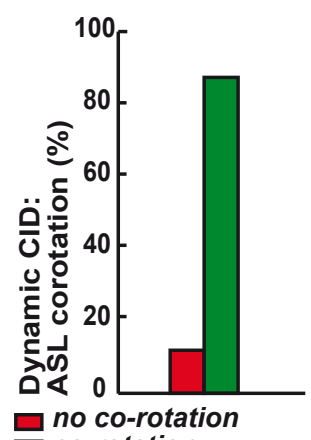

E

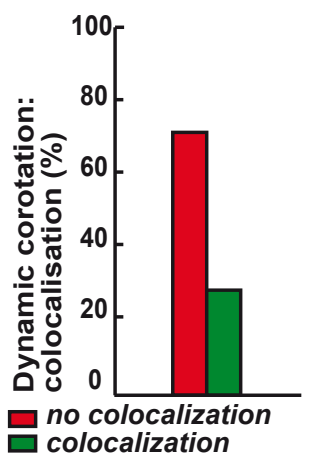

F

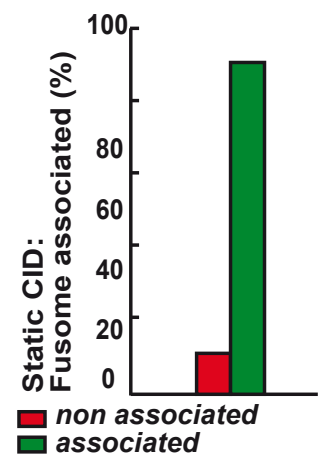

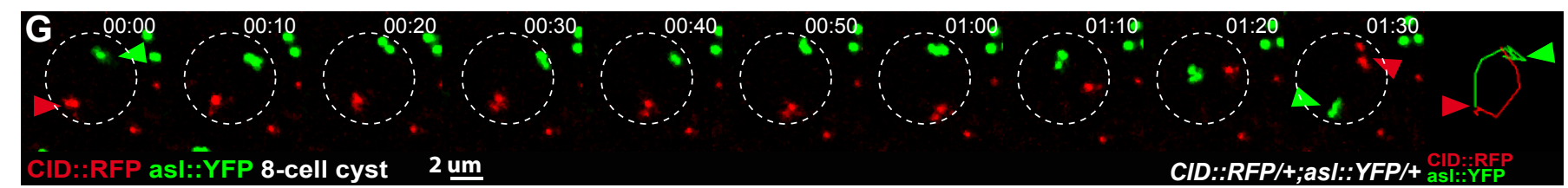
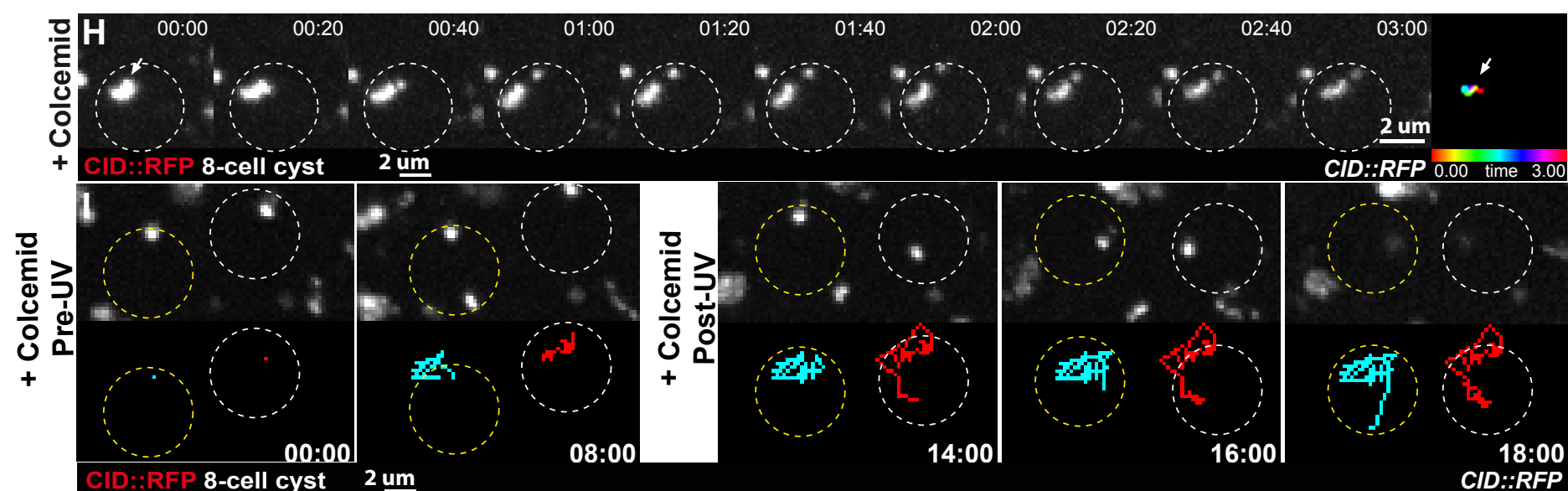

CID::RFP 0.00 time 3.00

J

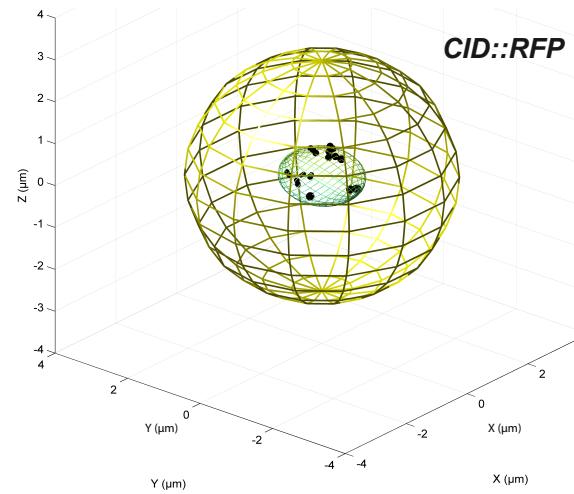

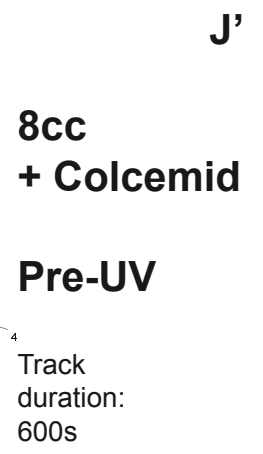

J'

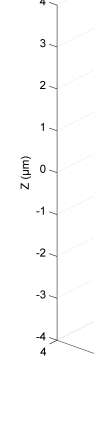

CID::RFP

$8 \mathrm{cc}$

+ Colcemid

Post-UV

Track

duration:

600 s

K

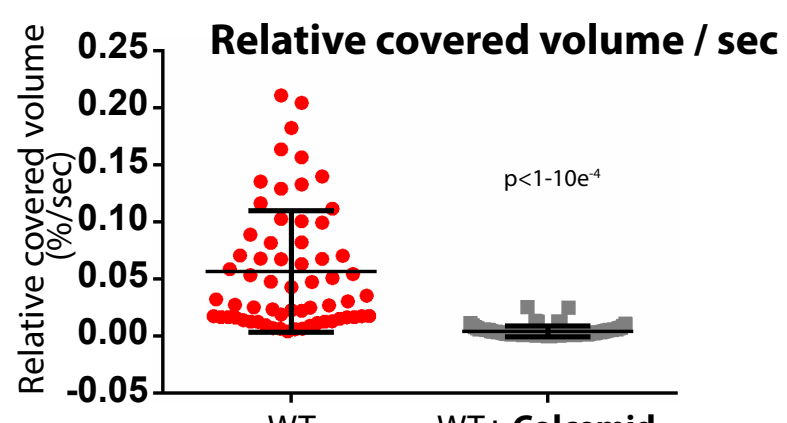




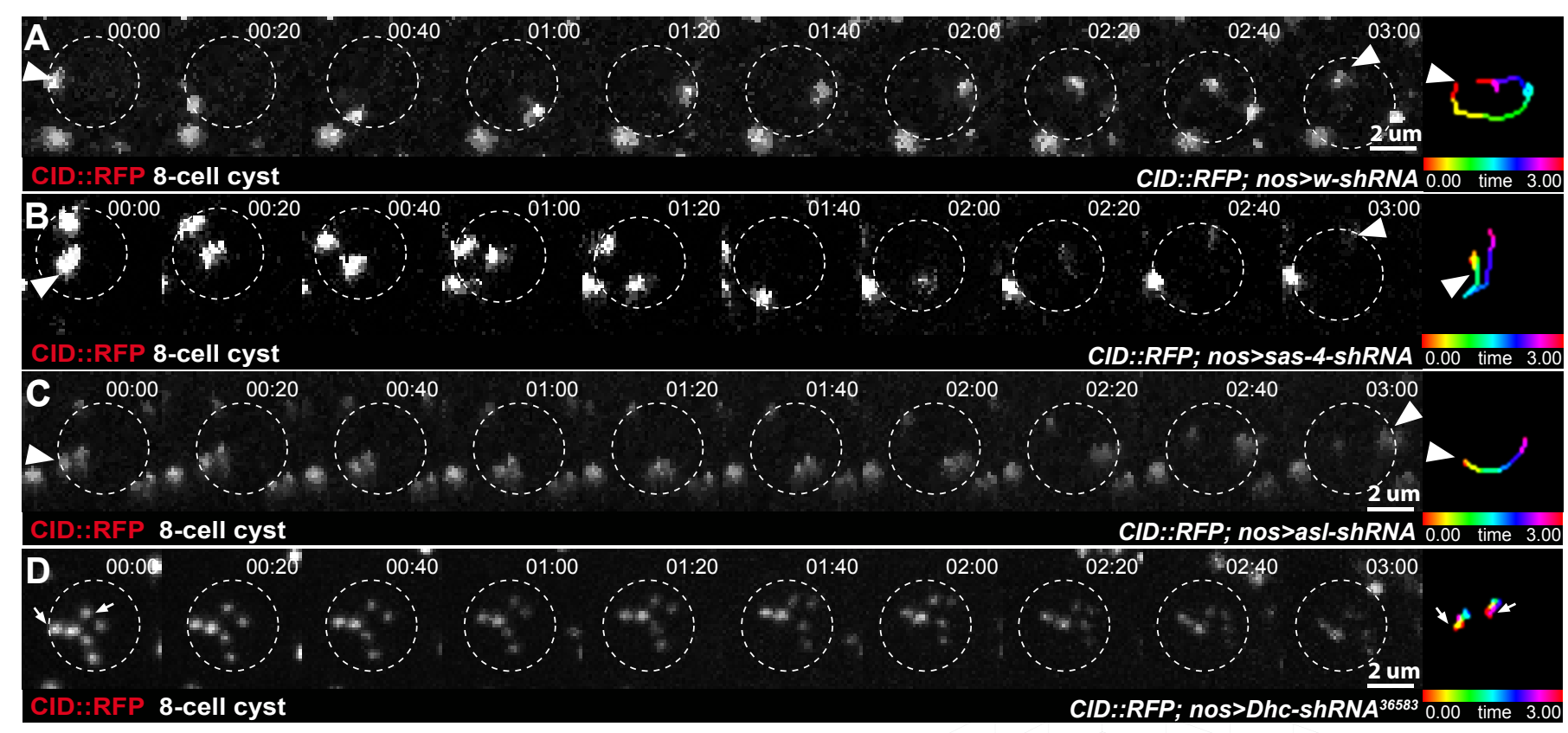

E

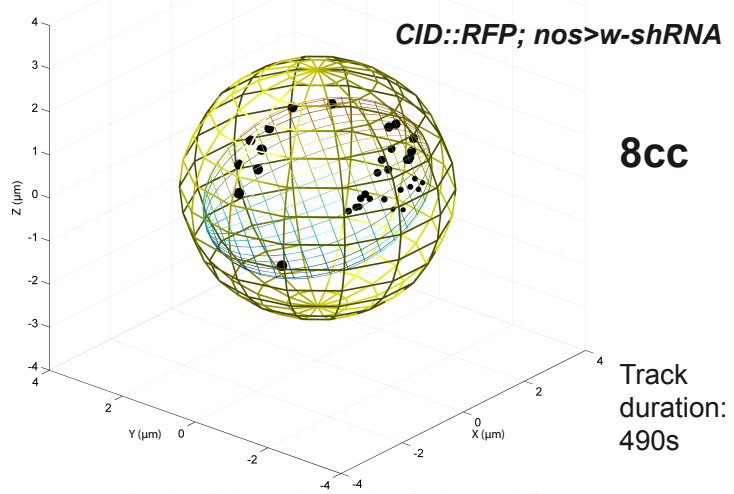

G

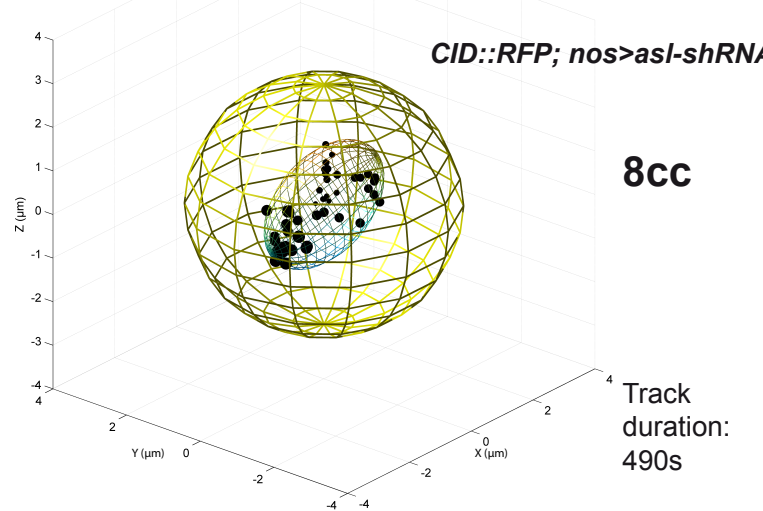

F

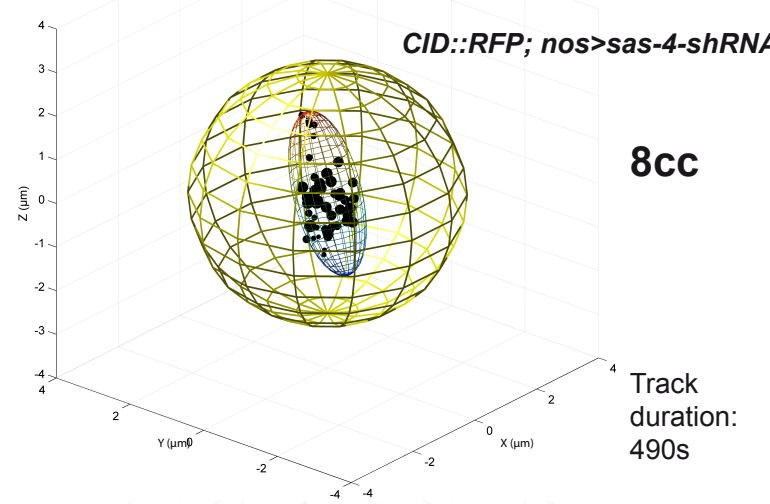

H

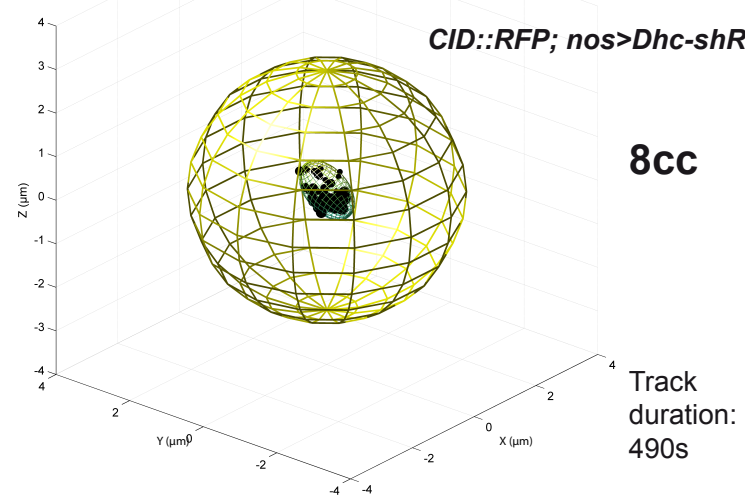

I

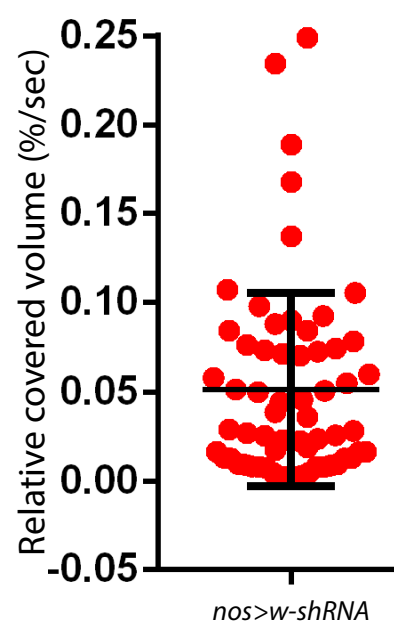

nos $>w-\operatorname{sh} R N A$

Relative covered volume / sec

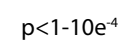

$p<1-10 e^{-4}$

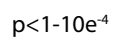

$\mathrm{p}<1-10 \mathrm{e}^{-4}$

$p<1-10 e^{-4}$
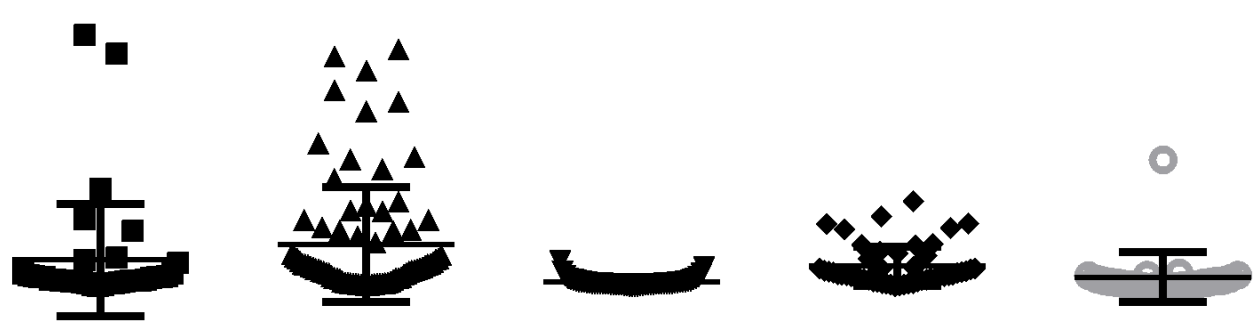

nos $>$ sas-4-shRNA

nos $>$ asl-shRNA

nos $>$ Dhc-shRNA $A^{36583}$

nos $>$ Dhc-shRNA 36698

Dhc- $64^{6-12} /{\text { Dhc- } 64^{3-2}}^{-2}$ 

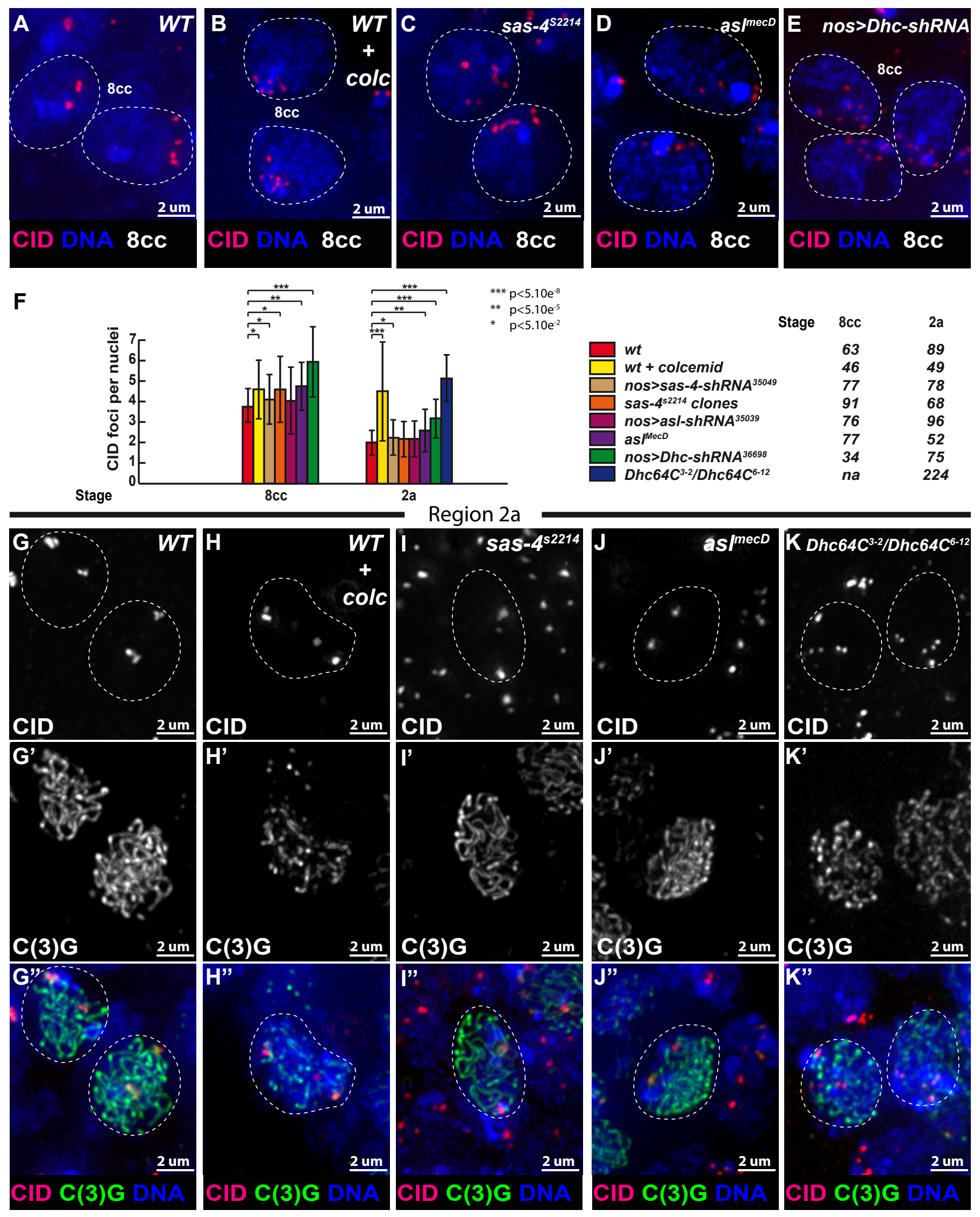

L

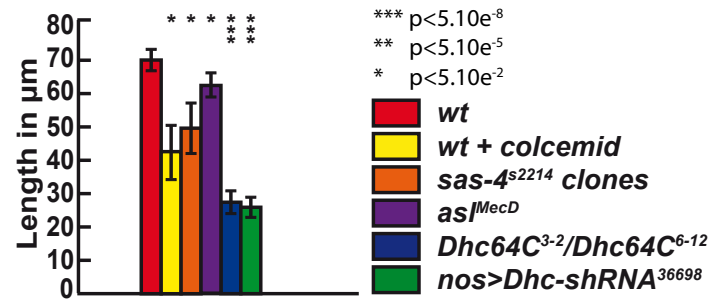



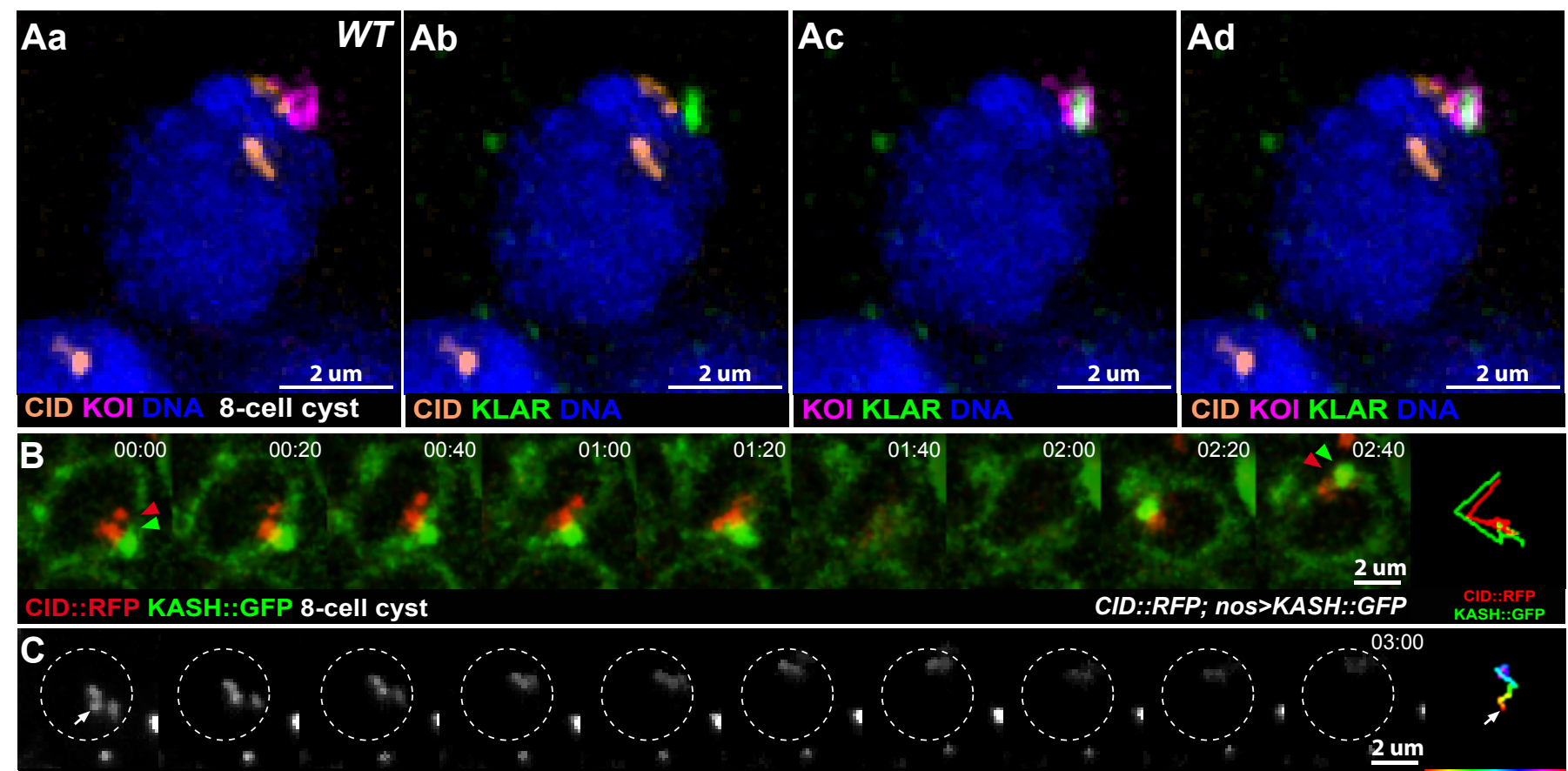

\section{CID::RFP 8-cell cyst}
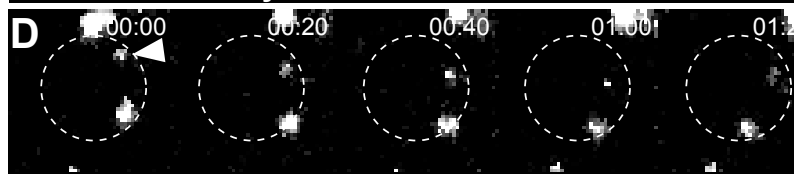

$0.1: 40$

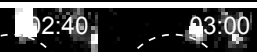

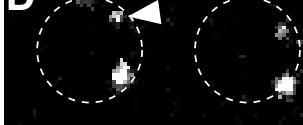

CID::RFP 8-cell cyst

4

E

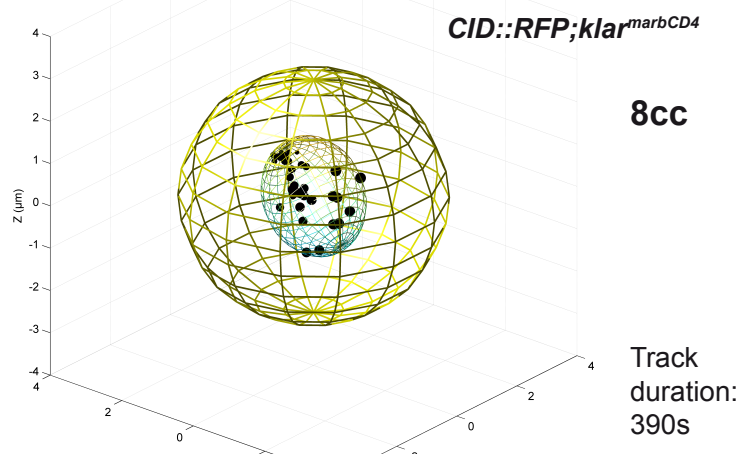

\section{G Relative covered volume / sec}

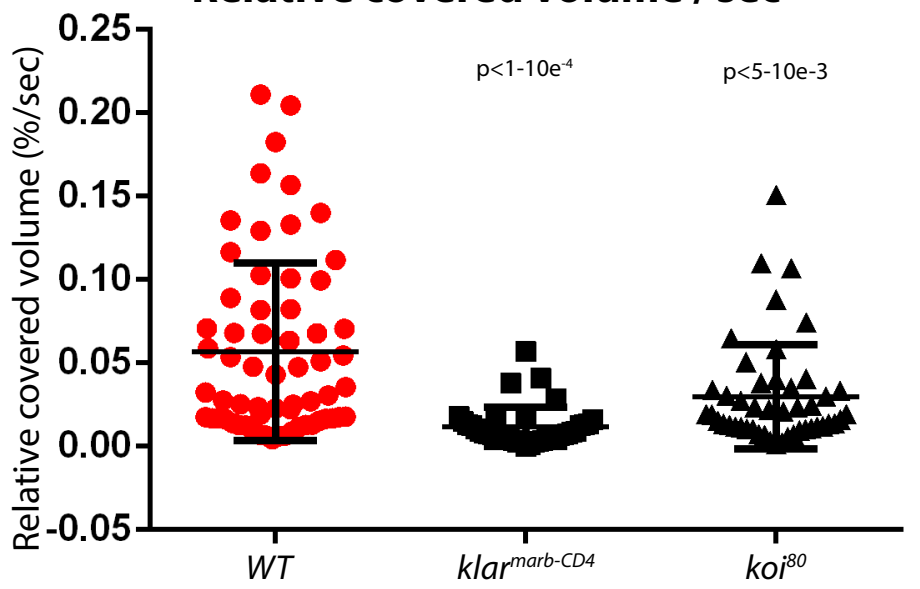

$\mathbf{F}$

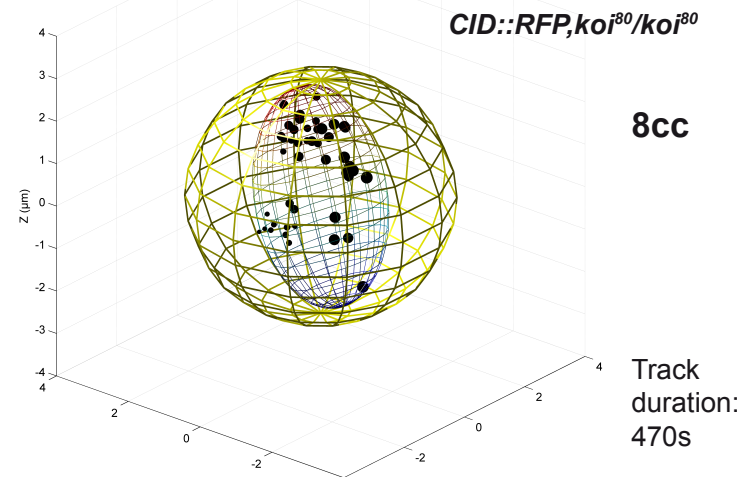

CID::RFP,koiso/koi ${ }^{80} 3.40$ time 6.40 

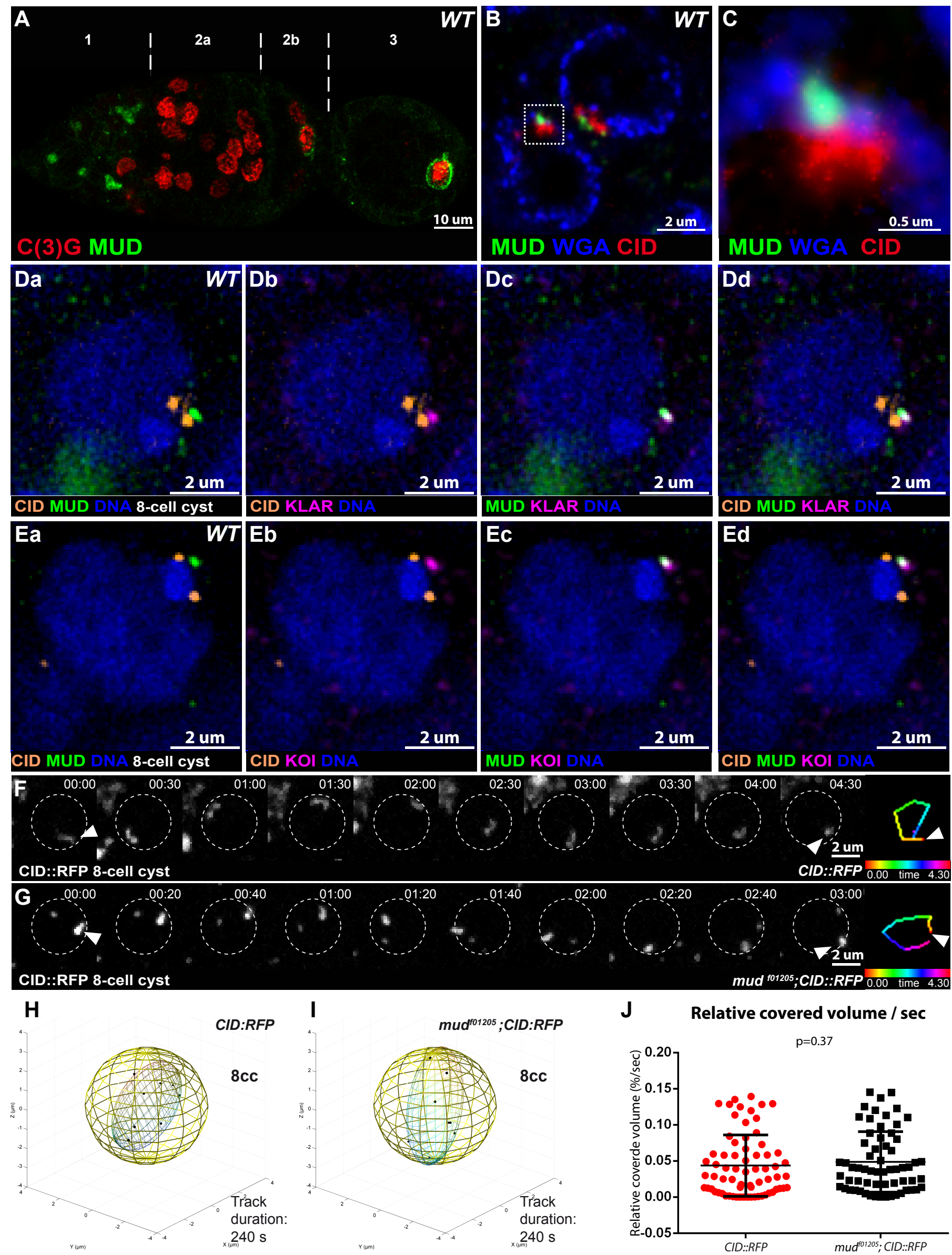

figure 7 


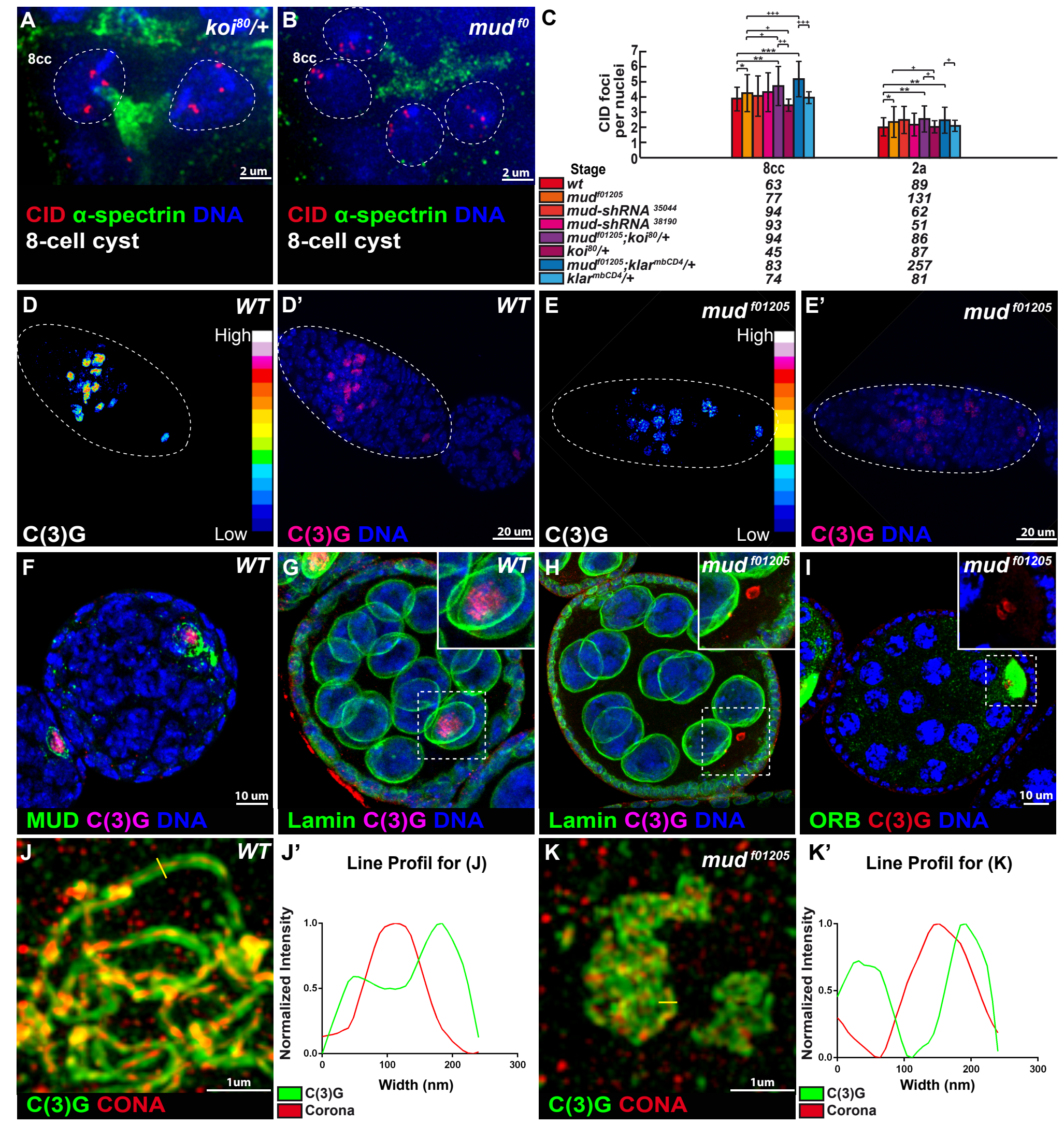

figure 8 